\title{
Minimax prediction of sequences with periodically stationary increments
}

\begin{abstract}
Kozak P.S. ${ }^{1}$, Luz M.M. ${ }^{2}$, Moklyachuk M.P. ${ }^{1, \bigotimes}$
The problem of optimal estimation of linear functionals constructed from unobserved values of a stochastic sequence with periodically stationary increments based on its observations at points $k<0$ is considered. For sequences with known spectral densities, we obtain formulas for calculating values of the mean square errors and the spectral characteristics of the optimal estimates of the functionals. Formulas that determine the least favourable spectral densities and minimax (robust) spectral characteristics of the optimal linear estimates of functionals are derived in the case where spectral densities of the sequence are not exactly known while some sets of admissible spectral densities are given.

Key words and phrases: periodically stationary increments, minimax-robust estimate, mean square error, least favourable spectral density, minimax spectral characteristic.
\end{abstract}

\footnotetext{
1 Taras Shevchenko National University of Kyiv, 64/13 Volodymyrska str., 01601, Kyiv, Ukraine

2 BNP Paribas Cardif, 8 Illinska str., 04070, Kyiv, Ukraine

$\bowtie$ Corresponding author

E-mail: petrokozak91@gmail.com(Kozak P.S.), maksym.luz@gmail.com (Luz M.M.), moklyachuk@gmail. com (Moklyachuk M.P.)
}

\section{Introduction}

The non-stationary and long memory time series models are of constant interest of researchers in the past decade (see, for example, papers by A. Dudek, H. Hurd and W. Wojtowicz [6], S. Johansen and M.O. Nielsen [13], V.A. Reisen et al. [33]). These models are used when analyzing data which arise in different field of economics, finance, climatology, air pollution, signal processing.

Since the first edition of the book by G.E.P. Box and G.M. Jenkins [4], autoregressive moving average (ARMA) models integrated of order $d$ are standard tool for time series analysis. These models are described by the equation

$$
\psi(B)(1-B)^{d} x_{t}=\theta(B) \varepsilon_{t} .
$$

where $\varepsilon_{t}, t \in \mathbb{Z}$, are zero mean i.i.d. random variables, $\psi(z), \theta(z)$ are polynomials of $p$ and $q$ degrees respectively with roots outside the unit circle. This integrated ARIMA model is generalized by adding a seasonal component. A new model is described by the equation (see [3] for details)

$$
\Psi\left(B^{s}\right)\left(1-B^{s}\right)^{D} x_{t}=\Theta\left(B^{s}\right) \varepsilon_{t},
$$

where $\Psi(z)$ and $\Theta(z)$ are polynomials of degrees of $P$ and $Q$ respectively which have roots outside the unit circle. 
When the ARIMA sequence determined by equation (1) is inserted into (2) instead of $\varepsilon_{t}$ we have a general multiplicative model $\Psi\left(B^{s}\right) \psi(B)(1-B)^{d}\left(1-B^{s}\right)^{D} x_{t}=\Theta\left(B^{s}\right) \theta(B) \varepsilon_{t}$ with parameters $(p, d, q) \times(P, D, Q)_{s}, d, D \in \mathbb{N}^{*}$, called SARIMA $(p, d, q) \times(P, D, Q)_{s}$ model.

A good performance is shown by models which include a fractional integration, that is when parameters $d$ and $D$ are fractional. We refer to the paper by S. Porter-Hudak [32] who studied a seasonal ARFIMA model and applied it to the monetary aggregates used by U.S. Federal Reserve.

Another type of non-stationarity is described by periodically correlated, or cyclostationary, processes introduced by E. G. Gladyshev [9]. These processes are widely used in signal processing and communications (see A. Napolitano [29] for a review of the recent works on cyclostationarity and its applications). Periodic time series may be considered as an extension of a SARIMA model (see R. Lund [19] for a test assessing if a PARMA model is preferable to a SARMA one) and are suitable for forecasting stream flows with quarterly, monthly or weekly cycles (see D. Osborn [30]). C. Baek, R.A. Davis and V. Pipiras [1] introduced a periodic dynamic factor model (PDFM) with periodic vector autoregressive (PVAR) factors, in contrast to seasonal VARIMA factors. I.V. Basawa, R. Lund and Q. Shao [2] investigated first-order seasonal autoregressive processes with periodically varying parameters.

The models mentioned above are used in estimation of model's parameters and forecast issues. Note, that direct application of the developed results to real data may lead to significant increasing of values of errors of estimates due to the presence of outliers, measurement errors, incomplete information about the spectral, or model structure etc. This is a reason of increasing interest to robust methods of estimation that are reasonable in such cases. For example, V.A. Reisen et al. [34] proposed a semiparametric robust estimator for the fractional parameters in the SARFIMA model and illustrated its application to forecasting of sulfur dioxide $\mathrm{SO}_{2}$ pollutant concentrations. C.C. Solci et al. [36] proposed robust estimates of periodic autoregressive (PAR) model. Robust approaches are successfully applied to the problem of estimation of linear functionals from unobserved values of stochastic processes. The paper by U. Grenander [10] should be marked as the first one where the minimax extrapolation problem for stationary processes was formulated as a game of two players and solved. Y. Hosoya [12], S.A. Kassam [15], S.A. Kassam and H.V. Poor [16], J. Franke [7], S. K. Vastola and H.V. Poor [37], M.P. Moklyachuk [23, 24] studied minimax extrapolation (forecasting), interpolation (missing values estimation) and filtering (smoothing) problems for the stationary sequences and processes. Recent results of minimax extrapolation problems for stationary vector processes and periodically correlated processes belong to M.P. Moklyachuk and A.Yu. Masyutka [25,26] and I.I. Dubovets'ka and M.P. Moklyachuk [5] respectively. Processes with stationary increments are investigated by M. Luz and M. Moklyachuk [20,21]. We also mention works by M.P. Moklyachuk and M.I. Sidei [27], O.Yu. Masyutka, M.P. Moklyachuk and M.I. Sidei [22,28], who derive minimax estimates of stationary processes from observations with missed values. P.S. Kozak and M.P. Moklyachuk [18] studied an interpolation problem for stochastic sequences with periodically stationary increments.

In this article we present results of investigation of the estimation problem for stochastic sequences with periodically stationary increments. In Section 1, we give definition of stochastic sequences $\eta(m)$ with periodically stationary (periodically correlated) increments. These non-stationary stochastic sequences combine periodic structure of covariation functions of sequences as well as the integrating one. This section also contains a short review of the spectral 
theory of vector-valued stationary increment sequences. Section 2 deals with the classical estimation problem for linear functionals in the case where spectral structure of the sequence $\eta(m)$ is exactly known. Estimates are obtained by representing the sequence $\eta(m)$ as a vector sequence $\vec{\xi}(m)$ with stationary increments and applying the Hilbert space projection technique. In Section 3, we derive the minimax (robust) estimates in the case, where spectral densities of sequences are not exactly known while some sets of admissible spectral densities are specified. In Subsections 3.1-3.4 we describe relations which determine the least favourable spectral densities and the minimax spectral characteristics of the optimal estimates of linear functionals for some sets of admissible spectral densities which are generalizations of the corresponding sets of admissible spectral densities described in a survey article by S.A. Kassam and H.V. Poor [16] for the case of stationary stochastic processes.

\section{Stochastic sequences with periodically stationary increments}

In this section, we present a brief review of the spectral theory of stochastic sequences with periodically stationary $n$th increments.

Consider a stochastic sequence $\{\eta(m), m \in \mathbb{Z}\}$ defined on a probability space $(\Omega, \mathcal{F}, \mathbb{P})$. Denote by $B_{\mu}$ a backward shift operator with the step $\mu \in \mathbb{Z}$, such that $B_{\mu} \eta(m)=\eta(m-\mu)$; $B:=B_{1}$. We first recall definitions and spectral properties of stochastic sequences with stationary $n$th increments (for more details and references see, e.g., [21, pp. 1-8], [8, pp. 48-60, 261268], [39, pp. 390-430]). The corresponding results for stochastic processes with continuous time are described in the articles by M.S. Pinsker and A.M. Yaglom [31] and A.M. Yaglom [38].

Definition 1. For a given stochastic sequence $\{\eta(m), m \in \mathbb{Z}\}$, the sequence

$$
\eta^{(n)}(m, \mu)=\left(1-B_{\mu}\right)^{n} \eta(m)=\sum_{l=0}^{n}(-1)^{l}\left(\begin{array}{l}
n \\
l
\end{array}\right) \eta(m-l \mu), \quad\left(\begin{array}{l}
n \\
l
\end{array}\right)=\frac{n !}{l !(n-l) !},
$$

is called a stochastic $n$th increment sequence with the step $\mu \in \mathbb{Z}$.

The stochastic $n$th increment sequence $\eta^{(n)}(m, \mu)$ satisfies the following relations:

$$
\eta^{(n)}(m,-\mu)=(-1)^{n} \eta^{(n)}(m+n \mu, \mu), \quad \eta^{(n)}(m, k \mu)=\sum_{l=0}^{(k-1) n} A_{l} \eta^{(n)}(m-l \mu, \mu), \quad k \in \mathbb{N},
$$

where coefficients $\left\{A_{l}, l=0,1,2, \ldots,(k-1) n\right\}$ are determined by the representation

$$
\left(1+x+\ldots+x^{k-1}\right)^{n}=\sum_{l=0}^{(k-1) n} A_{l} x^{l} .
$$

Definition 2. The stochastic $n$th increment sequence $\eta^{(n)}(m, \mu)$ generated by a stochastic sequence $\{\eta(m), m \in \mathbb{Z}\}$ is wide sense stationary if the mathematical expectations

$$
\mathrm{E} \eta^{(n)}\left(m_{0}, \mu\right)=c^{(n)}(\mu), \quad \mathrm{E} \eta^{(n)}\left(m_{0}+m, \mu_{1}\right) \eta^{(n)}\left(m_{0}, \mu_{2}\right)=D^{(n)}\left(m, \mu_{1}, \mu_{2}\right)
$$

exist for all $m_{0}, \mu, m, \mu_{1}, \mu_{2}$ and do not depend on $m_{0}$. The function $c^{(n)}(\mu)$ is called the mean value of the $n$th increment sequence $\eta^{(n)}(m, \mu)$ and the function $D^{(n)}\left(m, \mu_{1}, \mu_{2}\right)$ is called the structural function of the stationary $n$th increment sequence (or structural function of $n$th order of the stochastic sequence $\{\eta(m), m \in \mathbb{Z}\})$. The stochastic sequence $\{\eta(m), m \in \mathbb{Z}\}$ which determines the stationary $n$th increment sequence $\eta^{(n)}(m, \mu)$ by formula (3) is called a stochastic sequence with stationary $n$th increments (or integrated sequence of order $n$ ). 
The following spectral representation of the structural function of the stationary stochastic $n$th increment sequence holds true (see, e.g., [8, pp. 48-60]).

Theorem 1. The mean value $c^{(n)}(\mu)$ and the structural function $D^{(n)}\left(m, \mu_{1}, \mu_{2}\right)$ of the stationary stochastic $n$th increment sequence $\eta^{(n)}(m, \mu)$ can be represented in the forms

$$
\begin{gathered}
c^{(n)}(\mu)=c \mu^{n}, \\
D^{(n)}\left(m ; \mu_{1}, \mu_{2}\right)=\int_{-\pi}^{\pi} e^{i \lambda m}\left(1-e^{-i \mu_{1} \lambda}\right)^{n}\left(1-e^{i \mu_{2} \lambda}\right)^{n} \frac{1}{\lambda^{2 n}} d F(\lambda),
\end{gathered}
$$

where $c$ is a constant, $F(\lambda)$ is a left-continuous nondecreasing bounded function such that $F(-\pi)=0$. The constant $c$ and the function $F(\lambda)$ are determined uniquely by the increment sequence $\eta^{(n)}(m, \mu)$. On the other hand, a function $c^{(n)}(\mu)$ which has form (4) with a constant $c$ and a function $D^{(n)}\left(m ; \mu_{1}, \mu_{2}\right)$ which has form (5) with a function $F(\lambda)$ which satisfies the indicated conditions are the mean value and the structural function of a stationary $n$th increment sequence $\eta^{(n)}(m, \mu)$.

Note, that we will call by spectral function and spectral density of the stochastic sequence with stationary increments the spectral function and the spectral density of the corresponding stationary increment sequence.

Making use of representation (5) and the Karhunen theorem (see [8, pp. 261-268], [14, Theorem 10]) one can obtain the spectral representation of the stationary $n$th increment sequence $\eta^{(n)}(m, \mu):$

$$
\eta^{(n)}(m, \mu)=\int_{-\pi}^{\pi} e^{i m \lambda}\left(1-e^{-i \mu \lambda}\right)^{n} \frac{1}{(i \lambda)^{n}} d Z_{\eta^{(n)}}(\lambda),
$$

where $Z_{\eta^{(n)}}(\lambda)$ is a stochastic process with uncorrelated increments on $[-\pi, \pi)$ connected with the spectral function $F(\lambda)$ by the relation $E\left|Z_{\eta^{(n)}}\left(\lambda_{2}\right)-Z_{\eta^{(n)}}\left(\lambda_{1}\right)\right|^{2}=F\left(\lambda_{2}\right)-F\left(\lambda_{1}\right)<\infty$, $-\pi \leq \lambda_{1}<\lambda_{2}<\pi$.

Definition 3. A stochastic sequence $\{\zeta(m), m \in \mathbb{Z}\}$ is called a stochastic sequence with periodically stationary (periodically correlated) increments with period $T$ if the $n$th increment sequence $\zeta^{(n)}(m, \mu T)=\left(1-B_{\mu T}\right)^{n} \zeta(m)$ is stationary.

It follows from Definition 3 that the sequence

$$
\xi_{p}(m)=\zeta(m T+p-1), \quad p=1,2, \ldots, T, \quad m \in \mathbb{Z},
$$

forms a vector-valued sequence $\vec{\xi}(m)=\left\{\xi_{p}(m)\right\}_{p=1,2, \ldots, T}, m \in \mathbb{Z}$, with stationary $n$th increments. Really, for all $p=1,2, \ldots, T$,

$$
\begin{aligned}
\xi_{p}^{(n)}(m, \mu) & =\sum_{l=0}^{n}(-1)^{l}\left(\begin{array}{l}
n \\
l
\end{array}\right) \xi_{p}(m-l \mu) \\
& =\sum_{l=0}^{n}(-1)^{l}\left(\begin{array}{l}
n \\
l
\end{array}\right) \zeta((m-l \mu) T+p-1)=\zeta^{(n)}(m T+p-1, \mu T),
\end{aligned}
$$

where $\xi_{p}^{(n)}(m, \mu)$ is the $n$th increment of the $p$ th component of the vector-valued sequence $\vec{\xi}(m)$ (for more details see [18]).

The following spectral representations of the structural function of the vector-valued stationary stochastic $n$th increment sequence and the increment sequence itself hold true (see, e.g., [8, pp. 48-60, 261-268]). 
Theorem 2. The structural function $D^{(n)}\left(m, \mu_{1}, \mu_{2}\right)$ of the vector-valued stochastic stationary $n$th increment sequence $\vec{\xi}^{(n)}(m, \mu)$ can be represented in the form

$$
D^{(n)}\left(m ; \mu_{1}, \mu_{2}\right)=\int_{-\pi}^{\pi} e^{i \lambda m}\left(1-e^{-i \mu_{1} \lambda}\right)^{n}\left(1-e^{i \mu_{2} \lambda}\right)^{n} \frac{1}{\lambda^{2 n}} d F(\lambda),
$$

where $F(\lambda)$ is the matrix-valued spectral function of the stationary stochastic sequence $\vec{\xi}^{(n)}(m, \mu)$. The stationary $n$th increment sequence $\vec{\xi}^{(n)}(m, \mu)$ admits the spectral representation

$$
\vec{\xi}^{(n)}(m, \mu)=\int_{-\pi}^{\pi} e^{i m \lambda}\left(1-e^{-i \mu \lambda}\right)^{n} \frac{1}{(i \lambda)^{n}} d \vec{Z}_{\xi^{(n)}}(\lambda),
$$

where $\vec{Z}_{\xi^{(n)}}(\lambda)=\left\{Z_{p}(\lambda)\right\}_{p=1}^{T}$ is a (vector-valued) stochastic process with uncorrelated increments on $[-\pi, \pi)$ connected with the spectral function $F(\lambda)$ by the relation

$$
\mathrm{E}\left(Z_{p}\left(\lambda_{2}\right)-Z_{p}\left(\lambda_{1}\right)\right)\left(\overline{Z_{q}\left(\lambda_{2}\right)-Z_{q}\left(\lambda_{1}\right)}\right)=F_{p q}\left(\lambda_{2}\right)-F_{p q}\left(\lambda_{1}\right), \quad-\pi \leq \lambda_{1}<\lambda_{2}<\pi .
$$

Denote by $H=L_{2}(\Omega, \mathcal{F}, \mathbb{P})$ the Hilbert space of random variables $\zeta$ with zero first moment, $\mathrm{E} \zeta=0$, finite second moment, $\mathrm{E}|\zeta|^{2}<\infty$, and the inner product $(\zeta, \eta)=\mathrm{E} \zeta \bar{\eta}$. Denote by $H\left(\vec{\xi}^{(n)}\right)$ the subspace of the space $H=L_{2}(\Omega, \mathcal{F}, \mathbb{P})$ generated by components $\xi_{p}^{(n)}(m, \mu), p=$ $1,2, \ldots, T, m \in \mathbb{Z}$, of the stationary stochastic $n$th increment sequence $\vec{\xi}^{(n)}=\left\{\xi_{p}^{(n)}(m, \mu)\right\}_{p=1}^{T}$, and denote by $H^{r}\left(\vec{\xi}^{(n)}\right)$ the subspace generated by components $\xi_{p}^{(n)}(m, \mu), p=1,2, \ldots, T$, $m \leqslant r, r \in \mathbb{Z}$. Let $S\left(\vec{\xi}^{(n)}\right)=\bigcap_{r \in \mathbb{Z}} H^{r}\left(\vec{\xi}^{(n)}\right)$.

Since the space $S\left(\vec{\xi}^{(n)}\right)$ is a subspace of the Hilbert space $H\left(\vec{\xi}^{(n)}\right)$, the space $H\left(\vec{\xi}^{(n)}\right)$ admits the decomposition

$$
H\left(\vec{\zeta}^{(n)}\right)=S\left(\vec{\xi}^{(n)}\right) \oplus R\left(\vec{\zeta}^{(n)}\right),
$$

where $R\left(\vec{\xi}^{(n)}\right)$ is the orthogonal complement of the subspace $S\left(\vec{\zeta}^{(n)}\right)$ in the space $H\left(\vec{\zeta}^{(n)}\right)$.

Definition 4. A wide sense stationary stochastic $n$th increment sequence $\vec{\xi}^{(n)}(m, \mu)=$ $\left\{\xi_{p}^{(n)}(m, \mu)\right\}_{p=1}^{T}$ is called regular if $H\left(\vec{\xi}^{(n)}\right)=R\left(\vec{\xi}^{(n)}\right)$. It is called singular if $H\left(\vec{\xi}^{(n)}\right)=S\left(\vec{\xi}^{(n)}\right)$.

Making use of the decomposition (7) and Definition 4 we can verify that the following theorem holds true (see [11, pp. 157-163]).

Theorem 3. A wide-sense stationary stochastic $n$th increment sequence $\vec{\xi}^{(n)}(m, \mu)=$ $\left\{\xi_{p}^{(n)}(m, \mu)\right\}_{p=1}^{T}$ admits a unique representation in the form

$$
\xi_{p}^{(n)}(m, \mu)=\xi_{S, p}^{(n)}(m, \mu)+\xi_{R, p}^{(n)}(m, \mu),
$$

where $\xi_{R, p}^{(n)}(m, \mu), p=1,2, \ldots, T$, is a regular stationary increment sequence and $\xi_{S, p}^{(n)}(m, \mu)$, $p=1,2, \ldots, T$, is a singular stationary increment sequence. Moreover, the increment sequences $\xi_{R, p}^{(n)}(m, \mu)$ and $\xi_{S, p}^{(n)}(k, \mu), p=1,2, \ldots, T$, are orthogonal for all $m, k \in \mathbb{Z}$.

The components of representation (8) are defined by the formulas

$$
\xi_{S, p}^{(n)}(m, \mu)=\mathrm{E}\left[\xi_{p}^{(n)}(m, \mu) \mid S\left(\vec{\xi}^{(n)}\right)\right], \quad \xi_{R, p}^{(n)}(m, \mu)=\xi_{p}^{(n)}(m, \mu)-\xi_{S, p}^{(n)}(m, \mu), \quad p=1,2, \ldots, T .
$$

Consider a stochastic sequence $\vec{\varepsilon}(u)=\left\{\varepsilon_{k}(u)\right\}_{k=1}^{q}, u \in \mathbb{Z}$ of uncorrelated random variables satisfying the conditions: $\mathrm{E} \varepsilon_{k}(u) \bar{\varepsilon}_{j}(v)=\delta_{k j} \delta_{u v}$, where $\delta_{k j}$ and $\delta_{u v}$ are Kronecker symbols, and 
$\mathrm{E}\left|\varepsilon_{k}(u)\right|^{2}=1, k=1,2, \ldots, q, u \in \mathbb{Z}$. Denote by $H^{r}(\vec{\varepsilon})$ the Hilbert space generated by elements $\left\{\varepsilon_{k}(u): k=1,2, \ldots, q, u \leq r\right\}$.

We will call the sequence $\vec{\varepsilon}(u)=\left\{\varepsilon_{k}(u)\right\}_{k=1}^{q}, u \in \mathbb{Z}$, innovation sequence of a regular stationary $n$th increment sequence $\vec{\xi}^{(n)}(m, \mu)$ if the condition $H^{r}\left(\vec{\xi}^{(n)}\right)=H^{r}(\vec{\varepsilon})$ holds true for all $r \in \mathbb{Z}$.

Since elements $\left\{\varepsilon_{k}(u): k=1,2, \ldots, q, u \leq r\right\}$ of the innovation sequence form an orthonormal basis in the space $H^{r}(\vec{\varepsilon})$ and $H^{r}\left(\vec{\xi}^{(n)}\right)=H^{r}(\vec{\varepsilon})$ the following theorem holds true (see [11, pp. 157-163]).

Theorem 4. A stochastic stationary increment sequence $\vec{\xi}^{(n)}(m, \mu)$ is regular if and only if there exists an innovation sequence $\vec{\varepsilon}(u)=\left\{\varepsilon_{k}(u)\right\}_{k=1}^{q}, u \in \mathbb{Z}$, and a sequence of matrix-valued functions $\varphi^{(n)}(k, \mu)=\left\{\varphi_{i j}^{(n)}(k, \mu)\right\}_{i=\overline{1, T}}^{j=\overline{1, q}}, k \geq 0$, such that $\sum_{k=0}^{\infty} \sum_{i=1}^{T} \sum_{j=1}^{q}\left|\varphi_{i j}^{(n)}(k, \mu)\right|^{2}<\infty$ and

$$
\vec{\xi}^{(n)}(m, \mu)=\sum_{k=0}^{\infty} \varphi^{(n)}(k, \mu) \vec{\varepsilon}(m-k) .
$$

Representation (9) is called a canonical moving average representation of the stochastic stationary increment sequence $\vec{\xi}^{(n)}(m, \mu)$.

If the stationary $n$th increment sequence $\xi^{(n)}(m, \mu)$ admits the canonical representation (9), then its spectral function $F(\lambda)$ has the spectral density function $f(\lambda)=\left\{f_{i j}(\lambda)\right\}_{i, j=1}^{T}$ admitting the canonical factorization (see, e.g., [11, pp. 157-163]) $f(\lambda)=\Phi\left(e^{-i \lambda}\right) \Phi^{*}\left(e^{-i \lambda}\right)$, where the function $\Phi(z)=\sum_{k=0}^{\infty} \varphi(k) z^{k}$ has analytic in the unit circle $\{z:|z| \leq 1\}$ components $\Phi_{i j}(z)=$ $\sum_{k=0}^{\infty} \varphi_{i j}(k) z^{k}, i=1,2, \ldots, T, j=1,2, \ldots, q$.

Define $\Phi_{\mu}(z)=\sum_{k=0}^{\infty} \varphi^{(n)}(k, \mu) z^{k}=\sum_{k=0}^{\infty} \varphi_{\mu}(k) z^{k}$, where $\varphi_{\mu}(k)=\varphi^{(n)}(k, \mu)$ are coefficients from the canonical representation (9). Then the following relation holds true:

$$
\Phi_{\mu}\left(e^{-i \lambda}\right) \Phi_{\mu}^{*}\left(e^{-i \lambda}\right)=\frac{\left|1-e^{-i \lambda \mu}\right|^{2 n}}{\lambda^{2 n}} f(\lambda)
$$

The one-sided moving average representation (9) and relation (10) are used for finding the mean square optimal estimate of unobserved values of sequences with $n$th stationary increments.

\section{Hilbert space projection method of extrapolation}

Consider a vector-valued stochastic sequence with stationary $n$th increments $\vec{\xi}(m)$ constructed from the sequence $\zeta(m)$ with the help of transformation (6). Let the stationary $n$th increment sequence $\vec{\xi}^{(n)}(m, \mu)=\left\{\xi_{p}^{(n)}(m, \mu)\right\}_{p=1}^{T}$ has an absolutely continuous spectral function $F(\lambda)$ and the spectral density $f(\lambda)=\left\{f_{i j}(\lambda)\right\}_{i, j=1}^{T}$. Without loss of generality we will assume that $\mathrm{E} \vec{\xi}^{(n)}(m, \mu)=0$ and $\mu>0$.

Consider the problem of mean square optimal linear estimation of the functionals $A \vec{\xi}=$ $\sum_{k=0}^{\infty}(\vec{a}(k))^{\top} \vec{\xi}(k), A_{N} \vec{\xi}=\sum_{k=0}^{N}(\vec{a}(k))^{\top} \vec{\xi}(k)$ which depend on the unobserved values of the stochastic sequence $\vec{\xi}(k)=\left\{\tilde{\zeta}_{p}(k)\right\}_{p=1}^{T}$ with stationary $n$th increments. Estimates are based on observations of the sequence $\vec{\xi}(k)$ at points $k=-1,-2, \ldots$ 
To solve the stated problem we will require fulfillment of the following conditions. Suppose that coefficients $\vec{a}(k)=\left\{a_{p}(k)\right\}_{p=1}^{T}, k \geq 0$, and the linear transformation $D^{\mu}$ to be defined in Lemma 1 satisfy the conditions

$$
\sum_{k=0}^{\infty}\|\vec{a}(k)\|<\infty, \sum_{k=0}^{\infty}(k+1)\|\vec{a}(k)\|^{2}<\infty, \sum_{k=0}^{\infty}\left\|\left(D^{\mu} \mathbf{a}\right)_{k}\right\|<\infty, \sum_{k=0}^{\infty}(k+1)\left\|\left(D^{\mu} \mathbf{a}\right)_{k}\right\|^{2}<\infty .
$$

Here $\|\cdot\|$ is the Euclidean norm: $\|\vec{a}(k)\|^{2}=\sum_{p=1}^{T}\left|a_{p}(k)\right|^{2}$ for $\vec{a}(k)=\left\{a_{p}(k)\right\}_{p=1}^{T}$ and $\left.\left\|\left(D^{\mu} \mathbf{a}\right)_{k}\right\|^{2}=\sum_{p=1}^{T} \mid\left(D^{\mu} \mathbf{a}_{p}\right)_{k}\right)\left.\right|^{2}$ for $\left.\left(D^{\mu} \mathbf{a}\right)_{k}=\left\{\left(D^{\mu} \mathbf{a}_{p}\right)_{k}\right)\right\}_{p=1}^{T}$ (see Lemma 1 for more details).

Let the spectral density $f(\lambda)$ satisfy the minimality condition

$$
\int_{-\pi}^{\pi} \operatorname{Tr}\left[\frac{\lambda^{2 n}}{\mid 1-e^{i \lambda \mu \mid 2 n}} f^{-1}(\lambda)\right] d \lambda<\infty
$$

This is the necessary and sufficient condition under which the mean square errors of the estimates of the functionals $A \vec{\xi}$ and $A_{N} \vec{\xi}$ are not equal to 0 .

The following lemma and corollary describe representations of the functionals $A \vec{\xi}$ and $A_{N} \vec{\xi}$ as sums of functionals with finite variances and functionals depending on the observed values of the sequence $\vec{\xi}(k)$ (see $[18,20]$ ).

Lemma 1. The functional $A \vec{\xi}$ admits the representation

$$
\begin{gathered}
A \vec{\xi}=B \vec{\xi}-V \vec{\xi}, \quad B \vec{\xi}=\sum_{k=0}^{\infty}(\vec{b}(k))^{\top} \vec{\xi}^{(n)}(k, \mu), \quad V \vec{\xi}=\sum_{k=-\mu n}^{-1}(\vec{v}(k))^{\top} \vec{\xi}(k), \\
v_{p}(k)=\sum_{l=[-k / \mu]^{\prime}}^{n}(-1)^{l}\left(\begin{array}{l}
n \\
l
\end{array}\right) b_{p}(l \mu+k), \quad p=1,2, \ldots, T, \quad k=-1,-2, \ldots,-\mu n, \\
b_{p}(k)=\sum_{m=k}^{\infty} a_{p}(m) d_{\mu}(m-k)=\left(D^{\mu} \mathbf{a}_{p}\right)_{k}, \quad p=1,2, \ldots, T, \quad k=0,1,2, \ldots,
\end{gathered}
$$

$\vec{v}(k)=\left(v_{1}(k), v_{2}(k), \ldots, v_{T}(k)\right)^{\top}, \vec{b}(k)=\left(b_{1}(k), b_{2}(k), \ldots, b_{T}(k)\right)^{\top}$, where by $[x]^{\prime}$ we denote the least integer number among the numbers that are grater than or equal to $x$, coefficients $\left\{d_{\mu}(k): k \geq 0\right\}$ are determined by the relationship $\sum_{k=0}^{\infty} d_{\mu}(k) x^{k}=\left(\sum_{j=0}^{\infty} x^{\mu j}\right)^{n}, D^{\mu}$ is a linear transformation in the space $\ell_{2}$ determined by the matrix with elements $D_{k, j^{\prime}}^{\mu}, k, j=0,1,2, \ldots$, such that $D_{k, j}^{\mu}=d_{\mu}(j-k)$ if $0 \leq k \leq j$ and $D_{k, j}^{\mu}=0$ for $0 \leq j<k, D^{\mu} \mathbf{a}=\left\{D^{\mu} \mathbf{a}_{p}\right\}_{p=1}^{T}$, $\mathbf{a}_{p}=\left(a_{p}(0), a_{p}(1), a_{p}(2), \ldots\right)^{\top}, p=1,2, \ldots, T$.

Corollary 1. The functional $A_{N} \vec{\xi}$ allows the representation $A_{N} \vec{\xi}=B_{N} \vec{\xi}-V_{N} \vec{\xi}$, where $B_{N} \vec{\xi}=$ $\sum_{k=0}^{N}\left(\vec{b}_{N}(k)\right)^{\top} \vec{\xi}(n)(k, \mu), V_{N} \vec{\xi}=\sum_{k=-\mu n}^{-1}\left(\vec{v}_{N}(k)\right)^{\top} \vec{\xi}(k)$, the coefficients $\left.\vec{v}_{N}(k)\right)=\left\{v_{N, p}(k)\right\}_{p=1}^{T}$, $k=-1,-2, \ldots,-\mu n$, and $\left.\vec{b}_{N}(k)\right)=\left\{b_{N, p}(k)\right\}_{p=1}^{T}, k=0,1, \ldots, N$, are calculated by the formulas

$$
\begin{gathered}
v_{N, p}(k)=\sum_{l=[-k / \mu]^{\prime}}^{\min \{[(N-k) / \mu], n\}}(-1)^{l}\left(\begin{array}{l}
n \\
l
\end{array}\right) b_{N, p}(l \mu+k), \quad k=-1,-2, \ldots,-\mu n, \\
b_{N, p}(k)=\sum_{m=k}^{N} a_{p}(m) d_{\mu}(m-k)=\left(D_{N}^{\mu} \mathbf{a}_{N, p}\right)_{k}, \quad k=0,1, \ldots, N,
\end{gathered}
$$

where $D_{N}^{\mu}$ is the linear transformation in the space $\ell_{2}$ determined by an infinite matrix with the entries $\left(D_{N}^{\mu}\right)_{k, j}=d_{\mu}(j-k)$ if $0 \leq k \leq j \leq N$, and $\left(D_{N}^{\mu}\right)_{k, j}=0$ if $j<k$ or $j, k>N$, $D_{N}^{\mu} \mathbf{a}_{N}=\left\{D_{N}^{\mu} \mathbf{a}_{N, p}\right\}_{p=1}^{T}, \mathbf{a}_{N, p}=\left(a_{p}(0), a_{p}(1), a_{p}(2), \ldots, a_{p}(N), 0, \ldots\right)^{\top}, p=1,2, \ldots, T$. 
Denote by $\Delta(f, \widehat{A} \vec{\xi}):=\mathrm{E}|A \vec{\xi}-\widehat{A} \vec{\xi}|^{2}$ the mean square error of the estimate $\widehat{A} \vec{\xi}$ of the functional $A \vec{\xi}$ and let $\Delta(f, \widehat{B} \vec{\xi}):=\mathrm{E}|B \vec{\xi}-\widehat{B} \vec{\xi}|^{2}$ denote the mean square error of the estimate $\widehat{B} \vec{\xi}$ of the functional $B \vec{\xi}$. Since the functional $V \vec{\xi}$ depends on the observed values $\vec{\xi}(k)$, $k=-1,-2, \ldots,-\mu n$, the following equalities hold true

$$
\begin{gathered}
\widehat{A} \vec{\xi}=\widehat{B} \vec{\xi}-V \vec{\xi}, \\
\Delta(f ; \widehat{A} \vec{\xi})=\mathrm{E}|A \vec{\xi}-\widehat{A} \vec{\xi}|^{2}=\mathrm{E}|B \vec{\xi}-V \vec{\xi}-\widehat{B} \vec{\xi}+V \vec{\xi}|^{2}=\mathrm{E}|B \vec{\xi}-\widehat{B} \vec{\xi}|^{2}=\Delta(f ; \widehat{B} \vec{\xi}) .
\end{gathered}
$$

Thus, it is sufficient to find an optimal linear estimate of the functional $B \vec{\xi}$ in order to find the optimal linear estimate of the functional $A \vec{\xi}$. This estimate can be found with the help of the Hilbert space projection method proposed by A.N. Kolmogorov (see [17, p. 228]).

Denote by $H^{0-}\left(\vec{\xi}^{(n)}\right)$ the closed linear subspace generated by the elements $\left\{\xi_{p}^{(n)}(k, \mu): p=\right.$ $1,2, \ldots, T, k=-1,-2,-3, \ldots\}$ of the Hilbert space $H=L_{2}(\Omega, \mathcal{F}, \mathbb{P})$.

Denote by $L_{2}^{0-}(f)$ the subspace of the Hilbert space $L_{2}(f)$ of vector-valued functions with the inner product

$$
\left\langle g_{1} ; g_{2}\right\rangle_{L_{2}}=\int_{-\pi}^{\pi}\left(g_{1}(\lambda)\right)^{\top} f(\lambda) \overline{g_{2}(\lambda)} d \lambda
$$

which is generated by the functions $e^{i \lambda k}\left(1-e^{-i \lambda \mu}\right)^{n} \delta_{l} /(i \lambda)^{n}, \delta_{l}=\left\{\delta_{l p}\right\}_{p=1}^{T}, l=1,2, \ldots, T$, $k=-1,-2,-3, \ldots$, where $\delta_{l p}$ is Kronecker symbol.

The relation

$$
\xi_{p}^{(n)}(k, \mu)=\int_{-\pi}^{\pi} e^{i \lambda k}\left(1-e^{-i \lambda \mu}\right)^{n} \frac{1}{(i \lambda)^{n}} d Z_{p}(\lambda), \quad p=1,2, \ldots, T,
$$

implies one-to-one correspondence between elements $\xi_{p}^{(n)}(k, \mu)$ of the space $H^{0-}\left(\vec{\xi}_{\mu}^{(n)}\right)$ and elements $e^{i \lambda k}\left(1-e^{-i \lambda \mu}\right)^{n}(i \lambda)^{-n}$ of the space $L_{2}^{0-}(f)$.

The functional $B \vec{\xi}$ allows the spectral representation

$$
B \vec{\xi}=\int_{-\pi}^{\pi}\left(\vec{B}_{\mu}\left(e^{i \lambda}\right)\right)^{\top} \frac{\left(1-e^{-i \mu \lambda}\right)^{n}}{(i \lambda)^{n}} d \vec{Z}_{\xi(n)}(\lambda)
$$

where $\vec{B}_{\mu}\left(e^{i \lambda}\right)=\sum_{k=0}^{\infty} \vec{b}(k) e^{i \lambda k}=\sum_{k=0}^{\infty}\left(D^{\mu} \mathbf{a}\right)_{k} e^{i \lambda k}$.

Relation (13) implies that every linear estimate $\widehat{A} \vec{\xi}$ of the functional $A \vec{\xi}$ allows the representation

$$
\widehat{A} \vec{\xi}=\int_{-\pi}^{\pi}\left(\vec{h}_{\mu}(\lambda)\right)^{\top} d \vec{Z}_{\xi^{(n)}}(\lambda)-\sum_{k=-\mu n}^{-1}\left(\vec{v}_{\mu}(k)\right)^{\top} \vec{\xi}(k),
$$

where $\vec{h}_{\mu}(\lambda)=\left\{h_{p}(\lambda)\right\}_{p=1}^{T}$ is the spectral characteristic of the estimate $\widehat{B} \vec{\xi}$, which can be found as a projection of the element $\vec{B}_{\mu}\left(e^{i \lambda}\right)\left(1-e^{-i \lambda \mu}\right)^{n} /(i \lambda)^{n}$ on the subspace $L_{2}^{0-}(f)$. This estimate is characterized by the following conditions:

$$
\begin{gathered}
\vec{h}_{\mu}(\lambda) \in L_{2}^{0-}(f), \\
\left(\vec{B}_{\mu}\left(e^{i \lambda}\right) \frac{\left(1-e^{-i \lambda \mu}\right)^{n}}{(i \lambda)^{n}}-\vec{h}_{\mu}(\lambda)\right) \perp L_{2}^{0-}(f) .
\end{gathered}
$$

From the condition (16) we obtain the following relation

$$
\int_{-\pi}^{\pi}\left(\vec{B}_{\mu}\left(e^{i \lambda}\right) \frac{\left(1-e^{-i \mu \lambda}\right)^{n}}{(i \lambda)^{n}}-\vec{h}_{\mu}(\lambda)\right)^{\top} f(\lambda) e^{-i k \lambda} \frac{\left(1-e^{i \mu \lambda}\right)^{n}}{(-i \lambda)^{n}} d \lambda=0
$$


which holds true for all $k \leqslant-1$.

Thus, the spectral characteristic of the estimate $\widehat{B} \vec{\xi}$ can be represented in the form

$$
\left(\vec{h}_{\mu}(\lambda)\right)^{\top}=\left(\vec{B}_{\mu}\left(e^{i \lambda}\right)\right)^{\top} \frac{\left(1-e^{-i \lambda \mu}\right)^{n}}{(i \lambda)^{n}}-\frac{(-i \lambda)^{n}\left(\vec{C}_{\mu}\left(e^{i \lambda}\right)\right)^{\top}}{\left(1-e^{i \lambda \mu}\right)^{n}} f^{-1}(\lambda), \quad \vec{C}_{\mu}\left(e^{i \lambda}\right)=\sum_{k=0}^{\infty} \vec{c}_{\mu}(k) e^{i k \lambda},
$$

where $\vec{c}(k)=\left\{c_{p}(k)\right\}_{p=1}^{T}, k \geqslant 0$, are unknown coefficients to be found.

Condition (15) implies that the spectral characteristic $\vec{h}_{\mu}(\lambda)$ can be presented as $\vec{h}_{\mu}(\lambda)=$ $\vec{h}(\lambda)\left(1-e^{-i \lambda \mu}\right)^{n} /(i \lambda)^{n}, \vec{h}(\lambda)=\sum_{k=1}^{\infty} \vec{s}(k) e^{-i \lambda k}$. The latter representation of the spectral characteristic $\vec{h}_{\mu}(\lambda)$ allows us to write the relations

$$
\int_{-\pi}^{\pi}\left[\left(\vec{B}_{\mu}\left(e^{i \lambda}\right)\right)^{\top}-\frac{\lambda^{2 n}\left(\vec{C}_{\mu}\left(e^{i \lambda}\right)\right)^{\top}}{\left(1-e^{-i \lambda \mu}\right)^{n}\left(1-e^{i \lambda \mu}\right)^{n}} f^{-1}(\lambda)\right] e^{-i j \lambda} d \lambda=0, \quad j \geqslant 0 .
$$

Next we define the Fourier coefficients of the function $\lambda^{2 n}\left|1-e^{i \lambda \mu}\right|^{-2 n} f^{-1}(\lambda)$ :

$$
F_{k, j}^{\mu}=\frac{1}{2 \pi} \int_{-\pi}^{\pi} e^{i \lambda(j-k)} \frac{\lambda^{2 n}}{\mid 1-e^{i \lambda \mu \mid 2 n}} f^{-1}(\lambda) d \lambda, \quad k, j \geq 0 .
$$

Making use of the defined Fourier coefficients, relation (18) can be presented as a system of linear equations $\vec{b}_{\mu}(j)=\sum_{k=0}^{\infty} F_{j, k}^{\mu} \vec{c}_{\mu}(k), j \geq 0$, determining the unknown coefficients $\vec{c}_{\mu}(k)$, $k \geq 0$.

Rewrite this system in the matrix form

$$
D^{\mu} \mathbf{a}=\mathbf{F}_{\mu} \mathbf{c}_{\mu},
$$

where $\mathbf{c}_{\mu}=\left(\left(\vec{c}_{\mu}(0)\right)^{\top},\left(\vec{c}_{\mu}(1)\right)^{\top},\left(\vec{c}_{\mu}(2)\right)^{\top}, \ldots\right)^{\top}$, a $=\left((\vec{a}(0))^{\top},(\vec{a}(1))^{\top},(\vec{a}(2))^{\top}, \ldots\right)^{\top}, \mathbf{F}_{\mu}$ is a linear operator in the space $\ell_{2}$, which is determined by a matrix with the $T \times T$ matrix entries $\left(\mathbf{F}_{\mu}\right)_{l, k}=F_{l, k}^{\mu}, l, k \geq 0$, the linear transformation $D^{\mu}$ is defined in Lemma 1.

To show that the operator $\mathbf{F}_{\mu}$ is invertible we note that the problem of projection of the element $B \vec{\xi}$ of the Hilbert space $H$ on the closed convex set $H^{0-}\left(\vec{\xi}_{\mu}^{(n)}\right)$ has a unique solution for each non-zero coefficients $\{\vec{a}(0), \vec{a}(1)), \vec{a}(2), \ldots\}$, satisfying conditions (11). Therefore, equation (19) has a unique solution for each vector $D^{\mu} \mathbf{a}$, which implies existence of the inverse operator $\mathbf{F}_{\mu}^{-1}$.

So, the coefficients $\vec{c}_{\mu}(k), k \geq 0$, which determine the spectral characteristic $\vec{h}_{\mu}(\lambda)$, can be calculated as $\vec{c}_{\mu}(k)=\left(\mathbf{F}_{\mu}^{-1} D^{\mu} \mathbf{a}\right)_{k}, k \geq 0$, where $\left(\mathbf{F}_{\mu}^{-1} D^{\mu} \mathbf{a}\right)_{k}, k \geq 0$, is the $k$ th $T$-dimension vector element of the vector $\mathbf{F}_{\mu}^{-1} D^{\mu} \mathbf{a}$.

The spectral characteristic $\vec{h}_{\mu}(\lambda)$ of the optimal estimate $\widehat{B} \vec{\xi}$ of the functional $B \vec{\xi}$ can be calculated by the formula

$$
\left(\vec{h}_{\mu}(\lambda)\right)^{\top}=\left(\vec{B}_{\mu}\left(e^{i \lambda}\right)\right)^{\top} \frac{\left(1-e^{-i \lambda \mu}\right)^{n}}{(i \lambda)^{n}}-\frac{(-i \lambda)^{n}\left(\sum_{k=0}^{\infty}\left(\mathbf{F}_{\mu}^{-1} D^{\mu} \mathbf{a}\right)_{k} e^{i k \lambda}\right)^{\top}}{\left(1-e^{i \lambda \mu}\right)^{n}} f^{-1}(\lambda) .
$$

The value of the mean square error of the estimate $\widehat{A} \vec{\xi}$ is calculated by the formula

$$
\begin{aligned}
\Delta(f ; \widehat{A} \vec{\xi}) & =\Delta(f ; \widehat{B} \vec{\xi})=\mathrm{E}|B \vec{\xi}-\widehat{B} \vec{\xi}|^{2} \\
& =\frac{1}{2 \pi} \int_{-\pi}^{\pi} \frac{(-i \lambda)^{n}\left(\sum_{k=0}^{\infty}\left(\mathbf{F}_{\mu}^{-1} D^{\mu} \mathbf{a}\right)_{k} e^{i k \lambda}\right)^{\top}}{\left(1-e^{i \lambda \mu}\right)^{n}} f(\lambda) \frac{(i \lambda)^{n}\left(\overline{\sum_{k=0}^{\infty}\left(\mathbf{F}_{\mu}^{-1} D^{\mu} \mathbf{a}\right)_{k} e^{i k \lambda}}\right)}{\left(1-e^{-i \lambda \mu}\right)^{n}} d \lambda \\
& =\left\langle D^{\mu} \mathbf{a}, \mathbf{F}_{\mu}^{-1} D^{\mu} \mathbf{a}\right\rangle .
\end{aligned}
$$


Next consider the case, where the spectral density $f(\lambda)=\left\{f_{i j}(\lambda)\right\}_{i, j=1}^{T}$ of the stochastic sequence $\vec{\xi}(m)$ admits the canonical factorization

$$
f(\lambda)=\Phi\left(e^{-i \lambda}\right) \Phi^{*}\left(e^{-i \lambda}\right), \quad \frac{\left|1-e^{-i \lambda \mu}\right|^{2 n}}{\lambda^{2 n}} f(\lambda)=\Phi_{\mu}\left(e^{-i \lambda}\right) \Phi_{\mu}^{*}\left(e^{-i \lambda}\right),
$$

where $\Phi\left(e^{-i \lambda}\right)=\sum_{k=0}^{\infty} \varphi(k) e^{-i k \lambda}, \Phi_{\mu}\left(e^{-i \lambda}\right)=\sum_{k=0}^{\infty} \varphi_{\mu}(k) e^{-i k \lambda}, \varphi_{\mu}(k)=\left\{\varphi_{i j}(k)\right\}_{i=\overline{1, T}}^{j=\overline{1, q}}, k=$ $0,1,2, \ldots$. Define the matrix-valued function $\Psi_{\mu}\left(e^{-i \lambda}\right)=\left\{\Psi_{i j}\left(e^{-i \lambda}\right)\right\}_{i=\overline{1, q}}^{j=\overline{1, T}}$ by the equation $\Psi_{\mu}\left(e^{-i \lambda}\right) \Phi_{\mu}\left(e^{-i \lambda}\right)=E_{q}$, where $E_{q}$ is the identity $q \times q$ matrix.

Formulas for calculation the spectral characteristic and the value of the mean square error can be presented in terms of the coefficients $\left\{\varphi_{\mu}(k): k=0,1,2, \ldots\right\}$. One can check that relation (17) is satisfied by the function

$$
\vec{h}_{\mu}(\lambda)=\frac{\left(1-e^{-i \lambda \mu}\right)^{n}}{(i \lambda)^{n}}\left(\vec{B}_{\mu}\left(e^{i \lambda}\right)-\left(\Psi_{\mu}\left(e^{-i \lambda}\right)\right)^{\top} \vec{r}_{\mu}\left(e^{i \lambda}\right)\right)
$$

where $\vec{r}_{\mu}\left(e^{i \lambda}\right)=\sum_{k=0}^{\infty}\left(D^{\mu} \mathbf{A} \varphi_{\mu}\right)_{k} e^{i \lambda k},\left(D^{\mu} \mathbf{A} \varphi_{\mu}\right)_{k}=\sum_{m=0}^{\infty} \sum_{l=k}^{\infty}\left(\varphi_{\mu}(m)\right)^{\top} \vec{a}(m+l) d_{\mu}(l-k)$, $\mathbf{A}$ is a linear symmetric operator, which is determined by the matrix with the entries $\mathbf{A}_{k, j}=\vec{a}(k+j)$, $k, j \geq 0$. Note that under the conditions (11) the operators $D^{\mu} \mathbf{A}$ and $\mathbf{A}$ are compact.

The value of the mean square error is calculated by the formula

$$
\begin{aligned}
\Delta(f ; \widehat{A} \vec{\xi}) & =\frac{1}{2 \pi} \int_{-\pi}^{\pi}\left(\sum_{k=0}^{\infty}\left(D^{\mu} \mathbf{A} \varphi_{\mu}\right)_{k} e^{i \lambda k}\right)^{\top}\left(\overline{\left.\sum_{k=0}^{\infty}\left(D^{\mu} \mathbf{A} \varphi_{\mu}\right)_{k} e^{i \lambda k}\right)} d \lambda\right. \\
& =\frac{1}{2 \pi} \int_{-\pi}^{\pi}\left\|\vec{r}_{\mu}\left(e^{i \lambda}\right)\right\|^{2} d \lambda=\left\|D^{\mu} \mathbf{A} \varphi_{\mu}\right\|^{2} .
\end{aligned}
$$

The derived results are summarized in the following theorem.

Theorem 5. Let a vector-valued stochastic sequence $\{\vec{\xi}(m), m \in \mathbb{Z}\}$ determine a stationary stochastic $n$th increment sequence $\vec{\xi}^{(n)}(m, \mu)$ with the spectral density matrix $f(\lambda)=$ $\left\{f_{i j}(\lambda)\right\}_{i, j=1}^{T}$, which satisfy the minimality condition (12). Let coefficients $\vec{a}(j), j \geqslant 0$, satisfy conditions (11). Then the optimal linear estimate $\widehat{A} \vec{\xi}$ of the functional $A \vec{\xi}$ based on observations of the sequence $\vec{\xi}(m)$ at points $m=-1,-2, \ldots$ is calculated by formula (14). The spectral characteristic $\vec{h}_{\mu}(\lambda)=\left\{h_{p}(\lambda)\right\}_{p=1}^{T}$ and the value of the mean square error $\Delta(f ; \widehat{A} \vec{\xi})$ of the optimal estimate $\widehat{A} \vec{\xi}$ are calculated by formulas (20) and (21) respectively. In the case, when the spectral density $f(\lambda)$ admits the canonical factorization (22), the spectral characteristic and the value of the mean square error of the optimal estimate $\widehat{A} \xi$ can be calculated by formulas (23) and (24) respectively.

Theorem 5 allows us to find the optimal estimate $\widehat{A}_{N} \vec{\xi}$ of the functional $A_{N} \vec{\xi}$, which depends on the unobserved values $\vec{\xi}(m), m=0,1,2, \ldots, N$, based on observations of the sequence $\vec{\xi}(m)$ at points $m=-1,-2, \ldots$ Put $\vec{a}(k)=0$ for $k>N$. Then we get that the spectral characteristic $\vec{h}_{\mu, N}(\lambda)$ of the optimal estimate

$$
\widehat{A}_{N} \vec{\xi}=\int_{-\pi}^{\pi}\left(\vec{h}_{\mu, N}(\lambda)\right)^{\top} d \vec{Z}_{\xi(n)}(\lambda)-\sum_{k=-\mu n}^{-1}\left(\vec{v}_{N}(k)\right)^{\top} \vec{\xi}(k)
$$


is calculated by the formula

$$
\left(\vec{h}_{\mu, N}(\lambda)\right)^{\top}=\left(\vec{B}_{\mu, N}\left(e^{i \lambda}\right)\right)^{\top} \frac{\left(1-e^{-i \lambda \mu}\right)^{n}}{(i \lambda)^{n}}-\frac{(-i \lambda)^{n}\left(\sum_{k=0}^{\infty}\left(\mathbf{F}_{\mu, N}^{-1} D_{N}^{\mu} \mathbf{a}_{N}\right)_{k} e^{i k \lambda}\right)^{\top}}{\left(1-e^{i \lambda \mu}\right)^{n}} f^{-1}(\lambda),
$$

where $B_{\mu, N}\left(e^{i \lambda}\right)=\sum_{k=0}^{N}\left(D_{N}^{\mu} \mathbf{a}_{N}\right)_{k} e^{i \lambda k}$, the linear transformation $D_{N}^{\mu}$ is defined in Corollary 1, $\mathbf{F}_{\mu, N}$ is a linear operator in the space $\ell_{2}$, which is determined by a matrix with the $T \times T$ matrix entries $\left(\mathbf{F}_{\mu, N}\right)_{l, m}=F_{l, m}^{\mu}, l \geq 0,0 \leq m \leq N$, and $\left(\mathbf{F}_{\mu, N}\right)_{l, m}=0, l \geq 0, m>N$. The value of the mean square error of the optimal estimate $\widehat{A}_{N} \xi$ is calculated by formula

$$
\begin{aligned}
\Delta\left(f, \widehat{A}_{N} \vec{\xi}\right) & =\Delta\left(f, \widehat{B}_{N} \vec{\xi}\right)=\mathrm{E}\left|B_{N} \vec{\xi}-\widehat{B}_{N} \vec{\xi}\right|^{2} \\
& =\frac{1}{2 \pi} \int_{-\pi}^{\pi} \frac{(-i \lambda)^{n}\left(\sum_{k=0}^{\infty}\left(\mathbf{F}_{\mu, N}^{-1} D_{N}^{\mu} \mathbf{a}_{N}\right)_{k} e^{i k \lambda}\right)^{\top}}{\left(1-e^{i \lambda \mu}\right)^{n}} f(\lambda) \frac{(i \lambda)^{n}\left(\overline{\sum_{k=0}^{\infty}\left(\mathbf{F}_{\mu, N}^{-1} D_{N}^{\mu} \mathbf{a}_{N}\right)_{k} e^{i k \lambda}}\right)}{\left(1-e^{-i \lambda \mu}\right)^{n}} d \lambda \\
& =\left\langle D_{N}^{\mu} \mathbf{a}_{N}, \mathbf{F}_{\mu, N}^{-1} D_{N}^{\mu} \mathbf{a}_{N}\right\rangle .
\end{aligned}
$$

In the case, when the spectral density $f(\lambda)$ admits the canonical factorization (22), the spectral characteristic can be calculated by the formula

$$
\begin{gathered}
\vec{h}_{\mu, N}(\lambda)=\frac{\left(1-e^{-i \lambda \mu}\right)^{n}}{(i \lambda)^{n}}\left(\vec{B}_{\mu, N}\left(e^{i \lambda}\right)-\left(\Psi_{\mu}\left(e^{-i \lambda}\right)\right)^{\top} \vec{r}_{\mu, N}\left(e^{i \lambda}\right)\right), \\
\vec{r}_{\mu, N}\left(e^{i \lambda}\right)=\sum_{k=0}^{N}\left(\widetilde{D}_{N}^{\mu} \mathbf{A}_{N} \varphi_{\mu, N}\right)_{k} e^{i k \lambda}, \quad\left(\widetilde{D}_{N}^{\mu} \mathbf{A}_{N} \varphi_{\mu, N}\right)_{k}=\sum_{m=0}^{N} \sum_{l=k}^{N}\left(\varphi_{\mu}(m)\right)^{\top} \vec{a}(m+l) d_{\mu}(l-k),
\end{gathered}
$$

where $\varphi_{\mu, N}=\left(\varphi_{\mu}(0), \varphi_{\mu}(1), \ldots, \varphi_{\mu}(N)\right), \mathbf{A}_{N}$ is a linear operator determined by the coefficients $\vec{a}(k), k=0,1, \ldots, N$, as follows: $\left(\mathbf{A}_{N}\right)_{k, j}=\vec{a}(k+j), 0 \leq k+j \leq N,\left(\mathbf{A}_{N}\right)_{k, j}=0, k+j>N$, $0 \leq k, j \leq N, \widetilde{D}_{N}^{\mu}$ is the matrix of the dimension $(N+1) \times(N+1)$, determined by the coefficients $\left(\widetilde{D}_{N}^{\mu}\right)_{k, j}=d_{\mu}(j-k)$ if $0 \leq k \leq j \leq N$ and $\left(\widetilde{D}_{N}^{\mu}\right)_{k, j}=0$ if $0 \leq j<k \leq N$.

The value of the mean square error is calculated by the formula

$$
\begin{aligned}
& \Delta\left(f ; \widehat{A}_{N} \vec{\xi}\right)=\frac{1}{2 \pi} \int_{-\pi}^{\pi}\left(\sum_{k=0}^{N}\left(\widetilde{D}_{N}^{\mu} \mathbf{A}_{N} \varphi_{\mu, N}\right)_{k} e^{i k \lambda}\right)^{\top} \overline{\left(\sum_{k=0}^{N}\left(\widetilde{D}_{N}^{\mu} \mathbf{A}_{N} \varphi_{\mu, N}\right)_{k} e^{i k \lambda}\right)} d \lambda \\
& =\frac{1}{2 \pi} \int_{-\pi}^{\pi}\left\|\vec{r}_{\mu, N}\left(e^{i \lambda}\right)\right\|^{2} d \lambda=\left\|\widetilde{D}_{N}^{\mu} \mathbf{A}_{N} \varphi_{\mu, N}\right\|^{2} .
\end{aligned}
$$

Thus, the following theorem holds true.

Theorem 6. Let $\{\vec{\xi}(m), m \in \mathbb{Z}\}$ be a stochastic sequence, which determine a stationary stochastic $n$th increment sequence $\vec{\xi}^{(n)}(m, \mu)$ with the spectral density matrix $f(\lambda)$, which satisfy the minimality condition (12). The optimal linear estimate $\widehat{A}_{N} \vec{\xi}$ of the functional $A_{N} \vec{\xi}$, based on observations of the sequence $\vec{\xi}(m)$ at points $m=-1,-2, \ldots$, is calculated by the formula (25). The spectral characteristic $\vec{h}_{\mu, N}(\lambda)=\left\{h_{\mu, N, p}(\lambda)\right\}_{p=1}^{T}$ and the value of the mean square error $\Delta\left(f ; \widehat{A}_{N} \vec{\xi}\right)$ are calculated by formulas (26) and (27) respectively. In the case, when the spectral density $f(\lambda)$ admits the canonical factorization (22), the spectral characteristic $\vec{h}_{\mu, N}(\lambda)$ and the value of the mean square error of the optimal estimate $\widehat{A}_{N} \vec{\xi}$ can be calculated by formulas (28) and (29) respectively. 
As a corollary from the proposed theorem, one can obtain the mean square optimal estimate of the unobserved value $A_{N, p} \vec{\xi}=\xi_{p}(N)=\vec{\xi}(N) \delta_{p}, p=1,2, \ldots, T, N \geq 0$, of the stochastic sequence with $n$th stationary increments based on observations of the sequence $\vec{\xi}(m)$ at points $m=-1,-2, \ldots$

Corollary 2. The optimal linear estimate $\widehat{\xi}_{p}(N)$ of the unobserved value $\xi_{p}(N), p=1, \ldots, T$, $N \geq 0$, of the stochastic sequence with $n$th stationary increments from observations of the sequence $\vec{\xi}(m)$ at points $m=-1,-2, \ldots$ is calculated by the formula

$$
\widehat{\xi}_{p}(N)=\int_{-\pi}^{\pi}\left(\vec{h}_{\mu, N, p}(\lambda)\right)^{\top} d \vec{Z}_{\xi^{(n)}}(\lambda)-\sum_{k=-\mu n}^{-1}\left(\vec{v}_{N}(k)\right)^{\top} \vec{\xi}(k) .
$$

The spectral characteristic $\vec{h}_{\mu, N, p}(\lambda)$ of the estimate is calculated by the formula

$$
\begin{aligned}
\left(\vec{h}_{\mu, N, p}(\lambda)\right)^{\top}= & \frac{\left(1-e^{-i \lambda \mu}\right)^{n}}{(i \lambda)^{n}}\left(\delta_{p} \sum_{k=0}^{N} d_{\mu}(N-k) e^{i \lambda k}\right)^{\top} \\
& -\frac{(-i \lambda)^{n}\left(\sum_{k=0}^{\infty}\left(\mathbf{F}_{\mu, N}^{-1} \mathbf{d}_{\mu, N}\right)_{k} e^{i k \lambda}\right)^{\top}}{\left(1-e^{i \lambda \mu}\right)^{n}} f^{-1}(\lambda),
\end{aligned}
$$

where $\mathbf{d}_{\mu, N}=\left(d_{\mu}(N), d_{\mu}(N-1), d_{\mu}(N-2), \ldots, d_{\mu}(0), 0, \ldots\right)^{\top}$. The value of the mean square error of the optimal estimate is calculated by the formula

$$
\begin{aligned}
\Delta\left(f ; \widehat{\xi}_{p}(N)\right) & =\Delta\left(f ; \widehat{\xi}_{p}^{(n)}(N, \mu)\right)=\mathrm{E}\left|\xi_{p}^{(n)}(N, \mu)-\widehat{\xi}_{p}^{(n)}(N, \mu)\right|^{2} \\
& =\frac{1}{2 \pi} \int_{-\pi}^{\pi} \frac{(-i \lambda)^{n}\left(\sum_{k=0}^{\infty}\left(\mathbf{F}_{\mu, N}^{-1} \mathbf{d}_{\mu, N}\right)_{k} e^{i k \lambda}\right)^{\top}}{\left(1-e^{i \lambda \mu}\right)^{n}} f(\lambda) \frac{(i \lambda)^{n}\left(\overline{\sum_{k=0}^{\infty}\left(\mathbf{F}_{\mu, N}^{-1} \mathbf{d}_{\mu, N}\right)_{k} e^{i k \lambda}}\right)}{\left(1-e^{-i \lambda \mu}\right)^{n}} d \lambda \\
& =\left\langle\mathbf{d}_{\mu, N}, \mathbf{F}_{\mu, N}^{-1} \mathbf{d}_{\mu, N}\right\rangle .
\end{aligned}
$$

In the case, when the spectral density $f(\lambda)$ admits the canonical factorization (22), the spectral characteristic and the value of the mean square error of the optimal estimate $\widehat{\xi}_{p}(N)$ can be calculated by the formulas

$$
\begin{aligned}
\vec{h}_{\mu, N, p}(\lambda)= & \frac{\left(1-e^{-i \lambda \mu}\right)^{n}}{(i \lambda)^{n}} e^{i N \lambda}\left[\delta_{p}-\left(\Psi_{\mu}\left(e^{-i \lambda}\right)\right)^{\top}\left(\sum_{k=0}^{N} \varphi_{\mu}(k) e^{-i \lambda k}\right)^{\top} \delta_{p}\right], \\
\Delta\left(f ; \widehat{\xi}_{p}(N)\right) & =\frac{1}{2 \pi} \int_{-\pi}^{\pi}\left[\left(\delta_{p}\right)^{\top} \sum_{k=0}^{N} \varphi_{\mu}(k) e^{-i \lambda k}\right]\left[\left(\delta_{p}\right)^{\top} \sum_{k=0}^{N} \varphi_{\mu}(k) e^{-i \lambda k}\right]^{*} d \lambda \\
& =\sum_{k=0}^{N} \sum_{j=1}^{q}\left|\varphi_{\mu, p, j}(k)\right|^{2} .
\end{aligned}
$$

Remark 1. Since for all $n \geq 1$ and $\mu \geq 1$ the condition

$$
\int_{-\pi}^{\pi}\left|\ln \frac{\left|1-e^{-i \lambda \mu}\right|^{2 n}}{\lambda^{2 n}}\right| d \lambda<\infty
$$

holds true, there exists a function $w_{\mu}(z)=\sum_{k=0}^{\infty} w_{\mu}(k) z^{k}, \sum_{k=0}^{\infty}\left|w_{\mu}(k)\right|^{2}<\infty$, such that (see [11, pp. 151-157]) $\left|1-e^{-i \lambda \mu}\right|^{2 n}=\lambda^{2 n}\left|w_{\mu}\left(e^{-i \lambda}\right)\right|^{2}$. In particular, the function $w_{\mu}(z)$ can be calculated by the formula

$$
w_{\mu}(z)=\exp \left\{\frac{1}{4 \pi} \int_{-\pi}^{\pi} \frac{e^{i \lambda}+z}{e^{i \lambda}-z} \ln \frac{\left|1-e^{-i \lambda \mu}\right|^{2 n}}{\lambda^{2 n}} d \lambda\right\}
$$


Then the following relation

$$
\Phi_{\mu}\left(e^{-i \lambda}\right)=w_{\mu}\left(e^{-i \lambda}\right) \Phi\left(e^{-i \lambda}\right) .
$$

holds true. Relation (35) implies the following relationship $\varphi_{\mu}(k)=\sum_{j=0}^{k} w_{\mu}(k-j) \varphi(j)$, $k=0,1, \ldots$, that is $\varphi_{\mu, i j}(k)=\sum_{m=0}^{k} w_{\mu}(k-m) \varphi_{i j}(m), i=1,2, \ldots, T, j=1,2, \ldots, q, k=0,1, \ldots$ This relation can be represented in the form $\varphi_{\mu}=\mathbf{W}^{\mu} \boldsymbol{\varphi}$, where $\varphi_{\mu}=\left(\varphi_{\mu}(0), \varphi_{\mu}(1), \varphi_{\mu}(2), \ldots\right)^{\top}$ and $\boldsymbol{\varphi}=(\varphi(0), \varphi(1), \varphi(2), \ldots)^{\top}$ are vectors composed from matrices $\varphi_{\mu}(k)=\left\{\varphi_{\mu, i j}(k)\right\}_{i=\overline{1, T^{\prime}}}^{j=\bar{q}}$ $k=0,1,2, \ldots$, and $\varphi(k)=\left\{\varphi_{i j}(k)\right\}_{i=\overline{1, T}}^{j=\overline{1, q}}, k=0,1,2, \ldots$, and where $\mathbf{W}^{\mu}$ is a linear operator determined by the matrix with elements $\left(\mathbf{W}^{\mu}\right)_{j, k}=w_{\mu}(j-k)$ if $0 \leq k \leq j$ and $\left(\mathbf{W}^{\mu}\right)_{j, k}=0$ if $0 \leq j<k$.

Consider the problem of mean square optimal linear estimation of the functionals $A \zeta=$ $\sum_{k=0}^{\infty} a^{(\zeta)}(k) \zeta(k), A_{M} \zeta=\sum_{k=0}^{N} a^{(\zeta)}(k) \zeta(k)$, which depend on unobserved values of a stochastic sequence $\zeta(k)$ with periodically stationary increments. Estimates are based on observations of the sequence $\zeta(k)$ at points $k=-1,-2, \ldots$

The functional $A \zeta$ can be represented in the form

$$
\begin{aligned}
A \zeta & =\sum_{k=0}^{\infty} a^{(\zeta)}(k) \zeta(k)=\sum_{m=0}^{\infty} \sum_{p=1}^{T} a^{(\zeta)}(m T+p-1) \zeta(m T+p-1) \\
& =\sum_{m=0}^{\infty} \sum_{p=1}^{T} a_{p}(m) \xi_{p}(m)=\sum_{m=0}^{\infty}(\vec{a}(m))^{\top} \vec{\xi}(m)=A \vec{\xi},
\end{aligned}
$$

where for $m \in \mathbb{Z}$

$$
\begin{gathered}
\vec{\xi}(m)=\left(\xi_{1}(m), \xi_{2}(m), \ldots, \xi_{T}(m)\right)^{\top}, \quad \xi_{p}(m)=\zeta(m T+p-1), \quad p=1,2, \ldots, T, \\
\vec{a}(m)=\left(a_{1}(m), a_{2}(m), \ldots, a_{T}(m)\right)^{\top}, \quad a_{p}(m)=a^{(\zeta)}(m T+p-1), \quad p=1,2, \ldots, T .
\end{gathered}
$$

Making use of the introduced notations and statements of Theorem 5 we can claim that the following theorem holds true.

Theorem 7. Let a stochastic sequence $\zeta(k)$ with periodically stationary increments generate by (36) a vector-valued stochastic sequence $\vec{\xi}(m)$, which determine a stationary stochastic $n$th increment sequence $\vec{\xi}^{(n)}(m, \mu)$ with the spectral density matrix $f(\lambda)=\left\{f_{i j}(\lambda)\right\}_{i, j=1}^{T}$ that satisfy the minimality condition (12). Let coefficients $\vec{a}(k), k \geqslant 0$, determined by formula (37), satisfy conditions (11). Then the optimal linear estimate $\widehat{A} \zeta$ of the functional $A \zeta$ based on observations of the sequence $\zeta(m)$ at points $m=-1,-2, \ldots$ is calculated by formula (14). The spectral characteristic $\vec{h}_{\mu}(\lambda)=\left\{h_{p}(\lambda)\right\}_{p=1}^{T}$ and the value of the mean square error $\Delta(f ; \widehat{A} \zeta)$ of the optimal estimate $\widehat{A} \zeta$ are calculated by formulas (20) and (21) respectively. In the case, when the spectral density matrix $f(\lambda)$ admits the canonical factorization (22), the spectral characteristic and the value of the mean square error of the optimal estimate $\widehat{A} \xi$ can be calculated by formulas (23) and (24) respectively.

The functional $A_{M} \zeta$ can be represented in the form

$$
\begin{aligned}
A_{M} \zeta & =\sum_{k=0}^{M} a^{(\zeta)}(k) \zeta(k)=\sum_{m=0}^{N} \sum_{p=1}^{T} a^{(\zeta)}(m T+p-1) \zeta(m T+p-1)= \\
& =\sum_{m=0}^{N} \sum_{p=1}^{T} a_{p}(m) \xi_{p}(m)=\sum_{m=0}^{N}(\vec{a}(m))^{\top} \vec{\xi}(m)=A_{N} \vec{\xi},
\end{aligned}
$$


where $N=[M / T], \vec{\xi}(m)$ is determined by formula (36), $\vec{a}(m)=\left(a_{1}(m), a_{2}(m), \ldots, a_{T}(m)\right)^{\top}$,

$$
\begin{gathered}
a_{p}(m)=a^{(\zeta)}(m T+p-1), \quad 0 \leq m \leq N, 1 \leq p \leq T, m T+p-1 \leq M \\
a_{p}(N)=0, \quad M+1 \leq N T+p-1 \leq(N+1) T-1,1 \leq p \leq T .
\end{gathered}
$$

Making use of the introduced notations and statements of Theorem 6 we can claim that the following theorem holds true.

Theorem 8. Let a stochastic sequence $\zeta(k)$ with periodically stationary increments generate by (36) a vector-valued stochastic sequence $\vec{\xi}(m)$, which determine a stationary stochastic $n$th increment sequence $\vec{\zeta}^{(n)}(m, \mu)$ with the spectral density matrix $f(\lambda)=\left\{f_{i j}(\lambda)\right\}_{i, j=1}^{T}$ that satisfy the minimality condition (12). Let coefficients $\vec{a}(k), k \geqslant 0$, be determined by formula (38). The optimal linear estimate $\widehat{A}_{M} \zeta$ of the functional $A_{M} \zeta=A_{N} \vec{\xi}$ based on observations of the sequence $\zeta(m)$ at points $m=-1,-2, \ldots$ is calculated by formula (25). The spectral characteristic $\vec{h}_{\mu, N}(\lambda)=\left\{h_{\mu, N, p}(\lambda)\right\}_{p=1}^{T}$ and the value of the mean square error $\Delta\left(f ; \widehat{A}_{M} \zeta\right)$ are calculated by formulas (26) and (27) respectively. In the case, when the spectral density matrix $f(\lambda)$ admits the canonical factorization (22), the spectral characteristic $\vec{h}_{\mu, N}(\lambda)$ and the value of the mean square error of the optimal estimate $\widehat{A}_{M} \zeta$ can be calculated by formulas (28) and (29) respectively.

As a corollary from the proposed theorem, one can obtain the mean square optimal estimate of the unobserved value $\zeta(M), M \geq 0$, of a stochastic sequence $\zeta(m)$ with periodically stationary increments based on observations of the sequence $\zeta(m)$ at points $m=-1,-2, \ldots$ Making use of the notations $\zeta(M)=\xi_{p}(N)=\vec{\xi}(N) \delta_{p}, N=[M / T], p=M+1-N T$, and the obtained results we can conclude that the following corollary holds true.

Corollary 3. Let a stochastic sequence $\zeta(k)$ with periodically stationary increments generate by formula (36) a vector-valued stochastic sequence $\vec{\xi}(m)$, which determine a stationary stochastic $n$th increment sequence $\vec{\xi}^{(n)}(m, \mu)$ with the spectral density matrix $f(\lambda)=\left\{f_{i j}(\lambda)\right\}_{i, j=1}^{T}$ that satisfy the minimality condition (12). The optimal linear estimate $\widehat{\zeta(M)}$ of the unobserved value $\zeta(M), M \geq 0$, of a stochastic sequence $\zeta(m)$ with periodically stationary increments based on observations of the sequence $\zeta(m)$ at points $m=-1,-2, \ldots$ is calculated by formula (30). The spectral characteristic $\vec{h}_{\mu, N, p}(\lambda)$ of the estimate is calculated by the formula (31). The value of the mean square error of the optimal estimate is calculated by the formula (32). In the case, when the spectral density $f(\lambda)$ admits the canonical factorization (22), the spectral characteristic and the value of the mean square error of the optimal estimate $\widehat{\zeta(M)}$ can be calculated by the formulas (33), (34).

\section{Minimax (robust) method of extrapolation}

Values of the mean square errors and the spectral characteristics of the optimal estimates of the functionals $A \vec{\xi}$ and $A_{N} \vec{\xi}$ depending on the unobserved values of a stochastic sequence $\vec{\xi}(m)$, which determine a stationary stochastic $n$th increment sequence $\vec{\xi}^{(n)}(m, \mu)$ with the spectral density matrix $f(\lambda)$, based on observations of the sequence $\vec{\xi}(m)$ at points $m=-1,-2, \ldots$, can be calculated by formulas (20), (21) and (26), (27) respectively, provided the spectral density $f(\lambda)$ of the stochastic sequence $\vec{\xi}(m)$ is exactly known. If the spectral density $f(\lambda)$ admits the canonical factorization (22), formulas (23), (24) and (28), (29) can be used 
for calculating values of the mean square errors and the spectral characteristics, respectively. However, in practical cases spectral densities of sequences usually are not exactly known. If in such cases a set $\mathcal{D}$ of admissible spectral densities is defined, the minimax method of estimation of functionals depending on unobserved values of stochastic sequences with stationary increments may be applied. This method consists of finding an estimate that minimizes the maximal values of the mean square errors for all spectral densities from a given class $\mathcal{D}$ of admissible spectral densities simultaneously.

Definition 5. For a given class of spectral densities $\mathcal{D}$ a spectral density $f_{0}(\lambda) \in \mathcal{D}$ is called the least favourable in $\mathcal{D}$ for the optimal linear estimation of the functional $A \vec{\xi}$ if the following relation $\Delta\left(f_{0}\right)=\Delta\left(h_{\mu}\left(f_{0}\right) ; f_{0}\right)=\max _{f \in \mathcal{D}} \Delta\left(h_{\mu}(f) ; f\right)$ holds true.

Definition 6. For a given class of spectral densities $\mathcal{D}$ a spectral characteristic $h^{0}(\lambda)$ of the optimal linear estimate of the functional $A \xi$ is called minimax-robust if the following conditions $h^{0}(\lambda) \in H_{\mathcal{D}}=\bigcap_{f \in \mathcal{D}} L_{2}^{0-}(f), \min _{h \in H_{\mathcal{D}}} \max _{f \in \mathcal{D}} \Delta(h ; f)=\max _{f \in \mathcal{D}} \Delta\left(h^{0} ; f\right)$ are satisfied.

Taking into account the introduced definitions and the derived relations we can verify that the following lemmas hold true.

Lemma 2. A spectral density $f_{0}(\lambda) \in \mathcal{D}$ satisfying the minimality condition (12) is the least favourable density in the class $\mathcal{D}$ for the optimal linear extrapolation of the functional $A \vec{\xi}$ based on observations of the sequence $\vec{\xi}(m)$ at points $m=-1,-2, \ldots$ if the operator $\mathbf{F}_{\mu}^{0}$, defined by the Fourier coefficients of the function $f_{0}^{-1}(\lambda) \lambda^{2 n} /\left|1-e^{i \lambda \mu}\right|^{2 n}$, determines a solution to the constrained optimization problem

$$
\max _{f \in \mathcal{D}}\left\langle D^{\mu} \mathbf{a}, \mathbf{F}_{\mu}^{-1} D^{\mu} \mathbf{a}\right\rangle=\left\langle D^{\mu} \mathbf{a},\left(\mathbf{F}_{\mu}^{0}\right)^{-1} D^{\mu} \mathbf{a}\right\rangle .
$$

The minimax spectral characteristic $h^{0}=h_{\mu}\left(f^{0}\right)$ is calculated by formula (20) if $h_{\mu}\left(f^{0}\right) \in H_{\mathcal{D}}$.

Lemma 3. A spectral density $f_{0}(\lambda) \in \mathcal{D}$, which admits the canonical factorization (22), is the least favourable density in the class $\mathcal{D}$ for the optimal linear extrapolation of the functional $A \vec{\xi}$ based on observations of the sequence $\vec{\xi}(m)$ at points $m=-1,-2, \ldots$ if the coefficients $\left\{\varphi^{0}(k): k \geq 0\right\}$ of the canonical factorization $f_{0}(\lambda)=\left(\sum_{k=0}^{\infty} \varphi^{0}(k) e^{-i \lambda k}\right)\left(\sum_{k=0}^{\infty} \varphi^{0}(k) e^{-i \lambda k}\right)^{*}$ of the spectral density $f^{0}(\lambda)$ determine a solution to the constrained optimization problem

$$
\left\|D^{\mu} \mathbf{A} \varphi_{\mu}\right\|^{2} \rightarrow \max , \quad f(\lambda)=\left(\sum_{k=0}^{\infty} \varphi(k) e^{-i \lambda k}\right)\left(\sum_{k=0}^{\infty} \varphi(k) e^{-i \lambda k}\right)^{*} \in \mathcal{D} .
$$

The minimax spectral characteristic $h^{0}=h_{\mu}\left(f_{0}\right)$ is calculated by formula (23) if $h_{\mu}\left(f^{0}\right) \in H_{\mathcal{D}}$.

Lemma 4. A spectral density $f_{0}(\lambda) \in \mathcal{D}$, which admits the canonical factorization (22), is the least favourable density in the class $\mathcal{D}$ for the optimal linear extrapolation of the functional $A_{N} \vec{\xi}$ based on observations of the sequence $\vec{\xi}(m)$ at points $m=-1,-2, \ldots$ if the coefficients $\left\{\varphi^{0}(k): k=0,1, \ldots, N\right\}$ from the canonical factorization $f_{0}(\lambda)=\left(\sum_{k=0}^{N} \varphi^{0}(k) e^{-i \lambda k}\right)$ $\times\left(\sum_{k=0}^{N} \varphi^{0}(k) e^{-i \lambda k}\right)^{*}$ of the spectral density $f_{0}(\lambda)$ determine a solution to the constrained optimization problem $\left\|D_{N}^{\mu} \mathbf{A}_{N} \varphi_{\mu, N}\right\|^{2} \rightarrow \max , f(\lambda)=\left(\sum_{k=0}^{N} \varphi(k) e^{-i \lambda k}\right)\left(\sum_{k=0}^{N} \varphi(k) e^{-i \lambda k}\right)^{*} \in \mathcal{D}$. The minimax spectral characteristic $h^{0}=h_{\mu}\left(f_{0}\right)$ is calculated by formula (28) if $h_{\mu, N}\left(f^{0}\right) \in H_{\mathcal{D}}$. 
For more detailed analysis of properties of the least favourable spectral densities and minimax-robust spectral characteristics we observe that the minimax spectral characteristic $h^{0}$ and the least favourable spectral density $f_{0}$ form a saddle point of the function $\Delta(h ; f)$ on the set $H_{\mathcal{D}} \times \mathcal{D}$. The saddle point inequalities $\Delta\left(h ; f_{0}\right) \geq \Delta\left(h^{0} ; f_{0}\right) \geq \Delta\left(h^{0} ; f\right) \forall f \in \mathcal{D}, \forall h \in H_{\mathcal{D}}$ hold true if $h^{0}=h_{\mu}\left(f_{0}\right), h_{\mu}\left(f_{0}\right) \in H_{\mathcal{D}}$ and $f_{0}$ is a solution of the constrained optimization problem

$$
\widetilde{\Delta}(f)=-\Delta\left(h_{\mu}\left(f_{0}\right) ; f\right) \rightarrow \inf , \quad f \in \mathcal{D},
$$

where the functional $\Delta\left(h_{\mu}\left(f_{0}\right) ; f\right)$ is calculated by the formula

$$
\begin{aligned}
\Delta\left(h_{\mu}\left(f_{0}\right) ; f\right)=\frac{1}{2 \pi} & \int_{-\pi}^{\pi} \frac{(-i \lambda)^{n}\left(\sum_{k=0}^{\infty}\left(\left(\mathbf{F}_{\mu}^{0}\right)^{-1} D^{\mu} \mathbf{a}\right)_{k} e^{i k \lambda}\right)^{\top}}{\left(1-e^{i \lambda \mu}\right)^{n}} f_{0}^{-1}(\lambda) f(\lambda) \\
& \times f_{0}^{-1}(\lambda) \frac{(i \lambda)^{n}\left(\frac{\sum_{k=0}^{\infty}\left(\left(\mathbf{F}_{\mu}^{0}\right)^{-1} D^{\mu} \mathbf{a}\right)_{k} e^{i k \lambda}}{\left(1-e^{-i \lambda \mu}\right)^{n}}\right.}{(1-\lambda}
\end{aligned}
$$

or by the formula

$$
\begin{aligned}
& \Delta\left(h_{\mu}\left(f_{0}\right) ; f\right)=\frac{1}{2 \pi} \int_{-\pi}^{\pi} \frac{\left(1-e^{-i \lambda \mu}\right)^{n}}{(i \lambda)^{n}}\left(\sum_{k=0}^{\infty}\left(D^{\mu} \mathbf{A} \varphi_{\mu}^{0}\right)_{k} e^{i \lambda k}\right)^{\top} \Psi_{\mu}^{0}\left(e^{-i \lambda}\right) \\
& \times f(\lambda)\left(\Psi_{\mu}^{0}\left(e^{-i \lambda}\right)\right)^{*} \frac{\left(1-e^{i \lambda \mu}\right)^{n}}{(-i \lambda)^{n}}\left(\overline{\left.\sum_{k=0}^{\infty}\left(D^{\mu} \mathbf{A} \varphi_{\mu}^{0}\right)_{k} e^{i \lambda k}\right)}\right) d \lambda
\end{aligned}
$$

in the case, when the spectral density admits the canonical factorization (22).

The constrained optimization problem (41) is equivalent to the unconstrained optimization problem $\Delta_{\mathcal{D}}(f)=\widetilde{\Delta}(f)+\delta(f \mid \mathcal{D}) \rightarrow$ inf, where $\delta(f \mid \mathcal{D})$ is the indicator function of the set $\mathcal{D}$, namely $\delta(f \mid \mathcal{D})=0$ if $f \in \mathcal{D}$ and $\delta(f \mid \mathcal{D})=+\infty$ if $f \notin \mathcal{D}$. A solution $f_{0}$ of the unconstrained optimization problem is characterized by the condition $0 \in \partial \Delta_{\mathcal{D}}\left(f_{0}\right)$, which is the necessary and sufficient condition under which a point $f_{0}$ belongs to the set of minimums of the convex functional $\Delta_{\mathcal{D}}(f)$ (see, e.g., [35, Chapter 6]). This condition makes it possible to find the least favourable spectral densities in some special classes of spectral densities $\mathcal{D}$.

Note, that the form of the functional $\widetilde{\Delta}(f)$ allows us to apply the Lagrange method of indefinite multipliers for investigating the constrained optimization problem (41). Therefore, the complexity of optimization problem is determined by the complexity of calculating subdifferentials of the indicator functions of sets of admissible spectral densities.

\subsection{Least favourable spectral density in classes with integral restrictions}

Consider the prediction problem for the functional $A \vec{\xi}$, which depends on unobserved values of a sequence $\vec{\xi}(m)$, with stationary increments based on observations of the sequence at points $m=-1,-2, \ldots$ under the condition that the sets of admissible spectral densities $\mathcal{D}_{0}^{k}$, $k=1,2,3,4$, are defined as follows:

$$
\begin{gathered}
\mathcal{D}_{0}^{1}=\left\{f(\lambda): \frac{1}{2 \pi} \int_{-\pi}^{\pi} \frac{\left|1-e^{i \lambda \mu}\right|^{2 n}}{|\lambda|^{2 n}} f(\lambda) d \lambda=P\right\}, \\
\mathcal{D}_{0}^{2}=\left\{f(\lambda): \frac{1}{2 \pi} \int_{-\pi}^{\pi} \frac{\left|1-e^{i \lambda \mu}\right|^{2 n}}{|\lambda|^{2 n}} \operatorname{Tr}[f(\lambda)] d \lambda=p\right\}, \\
\mathcal{D}_{0}^{3}=\left\{f(\lambda): \frac{1}{2 \pi} \int_{-\pi}^{\pi} \frac{\left|1-e^{i \lambda \mu}\right|^{2 n}}{|\lambda|^{2 n}} f_{k k}(\lambda) d \lambda=p_{k}, k=\overline{1, T}\right\}, \\
\mathcal{D}_{0}^{4}=\left\{f(\lambda): \frac{1}{2 \pi} \int_{-\pi}^{\pi} \frac{\left|1-e^{i \lambda \mu}\right|^{2 n}}{|\lambda|^{2 n}}\left\langle B_{1}, f(\lambda)\right\rangle d \lambda=p\right\},
\end{gathered}
$$


where $p, p_{k}, k=\overline{1, T}$ are given numbers, $P, B_{1}$, are given positive-definite Hermitian matrices.

Define $\vec{C}_{\mu}^{f 0}\left(e^{i \lambda}\right)=\sum_{k=0}^{\infty}\left(\left(\mathbf{F}_{\mu}^{0}\right)^{-1} D^{\mu} \mathbf{a}\right)_{k} e^{i k \lambda}$ and $\vec{r}_{\mu}^{0}\left(e^{i \lambda}\right)=\sum_{k=0}^{\infty}\left(D^{\mu} \mathbf{A} \varphi_{\mu}^{0}\right)_{k} e^{i \lambda k}$.

From the condition $0 \in \partial \Delta_{\mathcal{D}}\left(f_{0}\right)$ we find the following equations, which determine the least favourable spectral densities for these given sets of admissible spectral densities.

For the first set of admissible spectral densities $\mathcal{D}_{0}^{1}$, we derive equation

$$
\vec{C}_{\mu}^{f 0}\left(e^{i \lambda}\right)\left(\vec{C}_{\mu}^{f 0}\left(e^{i \lambda}\right)\right)^{*}=\frac{\left|1-e^{i \lambda \mu}\right|^{2 n}}{|\lambda|^{2 n}} f_{0}(\lambda) \vec{\alpha} \vec{\alpha}^{*} \frac{\left|1-e^{i \lambda \mu}\right|^{2 n}}{|\lambda|^{2 n}} f_{0}(\lambda),
$$

where $\vec{\alpha}$ is a vector of Lagrange multipliers.

For the second set of admissible spectral densities $\mathcal{D}_{0}^{2}$, we derive equation

$$
\vec{C}_{\mu}^{f 0}\left(e^{i \lambda}\right)\left(\vec{C}_{\mu}^{f 0}\left(e^{i \lambda}\right)\right)^{*}=\frac{\left|1-e^{i \lambda \mu}\right|^{2 n}}{|\lambda|^{2 n}} f_{0}(\lambda)\left\{\alpha_{k}^{2} \delta_{k l}\right\}_{k, l=1}^{T} \frac{\left|1-e^{i \lambda \mu}\right|^{2 n}}{|\lambda|^{2 n}} f_{0}(\lambda),
$$

where $\alpha_{k}^{2}$ are Lagrange multipliers, $\delta_{k l}$ are Kronecker symbols.

For the third set of admissible spectral densities $\mathcal{D}_{0}^{3}$, we derive equation

$$
\vec{C}_{\mu}^{f 0}\left(e^{i \lambda}\right)\left(\vec{C}_{\mu}^{f 0}\left(e^{i \lambda}\right)\right)^{*}=\alpha^{2} \frac{\left|1-e^{i \lambda \mu}\right|^{2 n}}{|\lambda|^{2 n}} f_{0}(\lambda) B_{1}^{\top} \frac{\left|1-e^{i \lambda \mu}\right|^{2 n}}{|\lambda|^{2 n}} f_{0}(\lambda),
$$

where $\alpha^{2}$ is a Lagrange multiplier.

For the fourth set of admissible spectral densities $\mathcal{D}_{0}^{4}$, we derive equation

$$
\vec{C}_{\mu}^{f 0}\left(e^{i \lambda}\right)\left(\vec{C}_{\mu}^{f 0}\left(e^{i \lambda}\right)\right)^{*}=\alpha^{2}\left(\frac{\left|1-e^{i \lambda \mu}\right|^{2 n}}{|\lambda|^{2 n}} f_{0}(\lambda)\right)^{2},
$$

where $\alpha^{2}$ is a Lagrange multiplier.

In the case, when the spectral density admits the canonical factorization (22), we have the following equations, correspondingly

$$
\begin{gathered}
\vec{r}_{\mu}^{0}\left(e^{i \lambda}\right)\left(\vec{r}_{\mu}^{0}\left(e^{i \lambda}\right)\right)^{*}=\left(\Phi_{\mu}^{0}\left(e^{-i \lambda}\right)\right)^{\top} \vec{\alpha} \vec{\alpha}^{*} \overline{\Phi_{\mu}^{0}\left(e^{-i \lambda}\right)}, \\
\vec{r}_{\mu}^{0}\left(e^{i \lambda}\right)\left(\vec{r}_{\mu}^{0}\left(e^{i \lambda}\right)\right)^{*}=\left(\Phi_{\mu}^{0}\left(e^{-i \lambda}\right)\right)^{\top}\left\{\alpha_{k}^{2} \delta_{k l}\right\}_{k, l=1}^{T} \overline{\Phi_{\mu}^{0}\left(e^{-i \lambda}\right)}, \\
\vec{r}_{\mu}^{0}\left(e^{i \lambda}\right)\left(\vec{r}_{\mu}^{0}\left(e^{i \lambda}\right)\right)^{*}=\alpha^{2}\left(\Phi_{\mu}^{0}\left(e^{-i \lambda}\right)\right)^{\top} B_{1}^{\top} \overline{\Phi_{\mu}^{0}\left(e^{-i \lambda}\right)}, \\
\vec{r}_{\mu}^{0}\left(e^{i \lambda}\right)\left(\vec{r}_{\mu}^{0}\left(e^{i \lambda}\right)\right)^{*}=\alpha^{2}\left(\Phi_{\mu}^{0}\left(e^{-i \lambda}\right)\right)^{\top} \overline{\Phi_{\mu}^{0}\left(e^{-i \lambda}\right)} .
\end{gathered}
$$

The following theorem holds true.

Theorem 9. The least favourable spectral densities $f_{0}(\lambda)$ in the classes $\mathcal{D}_{0}^{k}, k=1,2,3,4$, for the optimal linear extrapolation of the functional $A \vec{\xi}$ from observations of the sequence $\vec{\xi}(m)$ at points $m=-1,-2, \ldots$ are determined by the minimality condition (12), equations (42)(45) respectively, the constrained optimization problem (39) and restrictions on densities from the corresponding classes $\mathcal{D}_{0}^{k}, k=1,2,3,4$. The minimax-robust spectral characteristic of the optimal estimate of the functional $A \vec{\xi}$ is determined by the formula (20). In the case, when spectral densities admit the canonical factorization (22), the least favourable spectral densities $f_{0}(\lambda)$ are determined by the minimality condition (12), equations (46)-(49), respectively, the constrained optimization problem (40), and restrictions on densities from the corresponding classes $\mathcal{D}_{0}^{k}, k=1,2,3,4$. The minimax-robust spectral characteristic of the optimal estimate of the functional $A \vec{\xi}$ is determined by the formula (23). 


\subsection{Least favourable spectral density in classes with inequality restrictions}

Consider the prediction problem for the functional $A \vec{\xi}$, which depends on unobserved values of a sequence $\vec{\xi}(m)$, with stationary increments based on observations of the sequence at points $m=-1,-2, \ldots$ under the condition that the sets of admissible spectral densities $\mathcal{D}_{V}^{U^{k}}, k=1,2,3,4$, are defined as follows:

$$
\begin{gathered}
\mathcal{D}_{V}^{U^{1}}=\left\{f(\lambda): V(\lambda) \leq f(\lambda) \leq U(\lambda), \frac{1}{2 \pi} \int_{-\pi}^{\pi} \frac{\left|1-e^{i \lambda \mu}\right|^{2 n}}{|\lambda|^{2 n}} f(\lambda) d \lambda=Q\right\}, \\
\mathcal{D}_{V}^{U^{2}}=\left\{f(\lambda): \operatorname{Tr}[V(\lambda)] \leq \operatorname{Tr}[f(\lambda)] \leq \operatorname{Tr}[U(\lambda)], \frac{1}{2 \pi} \int_{-\pi}^{\pi} \frac{\left|1-e^{i \lambda \mu}\right|^{2 n}}{|\lambda|^{2 n}} \operatorname{Tr}[f(\lambda)] d \lambda=q\right\}, \\
\mathcal{D}_{V}^{U^{3}}=\left\{f(\lambda): v_{k k}(\lambda) \leq f_{k k}(\lambda) \leq u_{k k}(\lambda), \frac{1}{2 \pi} \int_{-\pi}^{\pi} \frac{\left|1-e^{i \lambda \mu}\right|^{2 n}}{|\lambda|^{2 n}} f_{k k}(\lambda) d \lambda=q_{k}, k=\overline{1, T}\right\}, \\
\mathcal{D}_{V}^{U^{4}}=\left\{f(\lambda):\left\langle B_{2}, V(\lambda)\right\rangle \leq\left\langle B_{2}, f(\lambda)\right\rangle \leq\left\langle B_{2}, U(\lambda)\right\rangle, \frac{1}{2 \pi} \int_{-\pi}^{\pi} \frac{\left|1-e^{i \lambda \mu}\right|^{2 n}}{|\lambda|^{2 n}}\left\langle B_{2}, f(\lambda)\right\rangle d \lambda=q\right\} .
\end{gathered}
$$

Here spectral densities $V(\lambda), U(\lambda)$ are known and fixed, $q, q_{k}, k=\overline{1, T}$ are given numbers, $Q$, $B_{2}$ are given positive definite Hermitian matrices.

From the condition $0 \in \partial \Delta_{\mathcal{D}}\left(f_{0}\right)$ we find the following equations, which determine the least favourable spectral densities for these given sets of admissible spectral densities.

For the first set of admissible spectral densities $\mathcal{D}_{V}^{U^{1}}$, we derive equation

$$
\vec{C}_{\mu}^{f 0}\left(e^{i \lambda}\right)\left(\vec{C}_{\mu}^{f 0}\left(e^{i \lambda}\right)\right)^{*}=\frac{\left|1-e^{i \lambda \mu}\right|^{2 n}}{|\lambda|^{2 n}} f_{0}(\lambda)\left(\vec{\beta} \vec{\beta}^{*}+\Gamma_{1}(\lambda)+\Gamma_{2}(\lambda)\right) \frac{\left|1-e^{i \lambda \mu}\right|^{2 n}}{|\lambda|^{2 n}} f_{0}(\lambda),
$$

where $\vec{\beta}$ is a vector of Lagrange multipliers, $\Gamma_{1}(\lambda) \leq 0$ and $\Gamma_{1}(\lambda)=0$ if $f_{0}(\lambda)>V(\lambda)$, $\Gamma_{2}(\lambda) \geq 0$ and $\Gamma_{2}(\lambda)=0$ if $f_{0}(\lambda)<U(\lambda)$.

For the second set of admissible spectral densities $\mathcal{D}_{V}^{U^{2}}$, we derive equation

$$
\vec{C}_{\mu}^{f 0}\left(e^{i \lambda}\right)\left(\vec{C}_{\mu}^{f 0}\left(e^{i \lambda}\right)\right)^{*}=\left(\beta^{2}+\gamma_{1}(\lambda)+\gamma_{2}(\lambda)\right)\left(\frac{\left|1-e^{i \lambda \mu}\right|^{2 n}}{|\lambda|^{2 n}} f_{0}(\lambda)\right)^{2},
$$

where $\beta^{2}$ is Lagrange multiplier, $\gamma_{1}(\lambda) \leq 0$ and $\gamma_{1}(\lambda)=0$ if $\operatorname{Tr}\left[f_{0}(\lambda)\right]>\operatorname{Tr}[V(\lambda)], \gamma_{2}(\lambda) \geq 0$ and $\gamma_{2}(\lambda)=0$ if $\operatorname{Tr}\left[f_{0}(\lambda)\right]<\operatorname{Tr}[U(\lambda)]$.

For the third set of admissible spectral densities $\mathcal{D}_{V}^{U^{3}}$, we derive equation

$$
\vec{C}_{\mu}^{f 0}\left(e^{i \lambda}\right)\left(\vec{C}_{\mu}^{f 0}\left(e^{i \lambda}\right)\right)^{*}=\frac{\left|1-e^{i \lambda \mu}\right|^{2 n}}{|\lambda|^{2 n}} f_{0}(\lambda)\left\{\left(\beta_{k}^{2}+\gamma_{1 k}(\lambda)+\gamma_{2 k}(\lambda)\right) \delta_{k l}\right\}_{k, l=1}^{T} \frac{\left|1-e^{i \lambda \mu}\right|^{2 n}}{|\lambda|^{2 n}} f_{0}(\lambda),
$$

where $\beta_{k}^{2}$ are Lagrange multipliers, $\delta_{k l}$ are Kronecker symbols, $\gamma_{1 k}(\lambda) \leq 0$ and $\gamma_{1 k}(\lambda)=0$ if $f_{k k}^{0}(\lambda)>v_{k k}(\lambda), \gamma_{2 k}(\lambda) \geq 0$ and $\gamma_{2 k}(\lambda)=0$ if $f_{k k}^{0}(\lambda)<u_{k k}(\lambda)$.

For the fourth set of admissible spectral densities $\mathcal{D}_{V}^{U^{4}}$, we derive equation

$$
\vec{C}_{\mu}^{f 0}\left(e^{i \lambda}\right)\left(\vec{C}_{\mu}^{f 0}\left(e^{i \lambda}\right)\right)^{*}=\left(\beta^{2}+\gamma_{1}^{\prime}(\lambda)+\gamma_{2}^{\prime}(\lambda)\right) \frac{\left|1-e^{i \lambda \mu}\right|^{2 n}}{|\lambda|^{2 n}} f_{0}(\lambda) B_{2}^{\top} \frac{\left|1-e^{i \lambda \mu}\right|^{2 n}}{|\lambda|^{2 n}} f_{0}(\lambda),
$$

where $\beta^{2}$ is Lagrange multiplier, $\gamma_{1}^{\prime}(\lambda) \leq 0$ and $\gamma_{1}^{\prime}(\lambda)=0$ if $\left\langle B_{2}, f_{0}(\lambda)\right\rangle>\left\langle B_{2}, V(\lambda)\right\rangle$, $\gamma_{2}^{\prime}(\lambda) \geq 0$ and $\gamma_{2}^{\prime}(\lambda)=0$ if $\left\langle B_{2}, f_{0}(\lambda)\right\rangle<\left\langle B_{2}, U(\lambda)\right\rangle$. 
In the case, when the spectral density admits the canonical factorization (22), we have the following equations, correspondingly

$$
\begin{gathered}
\vec{r}_{\mu}^{0}\left(e^{i \lambda}\right)\left(\vec{r}_{\mu}^{0}\left(e^{i \lambda}\right)\right)^{*}=\left(\Phi_{\mu}^{0}\left(e^{-i \lambda}\right)\right)^{\top}\left(\vec{\beta}^{*}+\Gamma_{1}(\lambda)+\Gamma_{2}(\lambda)\right) \overline{\Phi_{\mu}^{0}\left(e^{-i \lambda}\right)}, \\
\vec{r}_{\mu}^{0}\left(e^{i \lambda}\right)\left(\vec{r}_{\mu}^{0}\left(e^{i \lambda}\right)\right)^{*}=\left(\beta^{2}+\gamma_{1}(\lambda)+\gamma_{2}(\lambda)\right)\left(\Phi_{\mu}^{0}\left(e^{-i \lambda}\right)\right)^{\top} \overline{\Phi_{\mu}^{0}\left(e^{-i \lambda}\right)}, \\
\vec{r}_{\mu}^{0}\left(e^{i \lambda}\right)\left(\vec{r}_{\mu}^{0}\left(e^{i \lambda}\right)\right)^{*}=\left(\Phi_{\mu}^{0}\left(e^{-i \lambda}\right)\right)^{\top}\left\{\left(\beta_{k}^{2}+\gamma_{1 k}(\lambda)+\gamma_{2 k}(\lambda)\right) \delta_{k l}\right\}_{k, l=1}^{T} \overline{\Phi_{\mu}^{0}\left(e^{-i \lambda}\right)}, \\
\vec{r}_{\mu}^{0}\left(e^{i \lambda}\right)\left(\vec{r}_{\mu}^{0}\left(e^{i \lambda}\right)\right)^{*}=\left(\beta^{2}+\gamma_{1}^{\prime}(\lambda)+\gamma_{2}^{\prime}(\lambda)\right)\left(\Phi_{\mu}^{0}\left(e^{-i \lambda}\right)\right)^{\top} B_{2}^{\top} \overline{\Phi_{\mu}^{0}\left(e^{-i \lambda}\right) .}
\end{gathered}
$$

The following theorem holds true.

Theorem 10. The least favourable spectral densities $f_{0}(\lambda)$ in the classes $\mathcal{D}_{V}^{U^{k}}, k=1,2,3,4$, for the optimal linear extrapolation of the functional $A \vec{\xi}$ from observations of the sequence $\vec{\xi}(m)$ at points $m=-1,-2, \ldots$ are determined by the minimality condition (12), equations (50)-(53), respectively, the constrained optimization problem (39), and restrictions on densities from the corresponding classes $\mathcal{D}_{V}^{U^{k}}, k=1,2,3,4$. The minimax-robust spectral characteristic of the optimal estimate of the functional $A \vec{\xi}$ is determined by the formula (20). In the case, when spectral densities admit the canonical factorization (22), the least favourable spectral densities $f_{0}(\lambda)$ are determined by the minimality condition (12), equations (54)-(57), respectively, the constrained optimization problem (40) and restrictions on densities from the corresponding $\mathcal{D}_{V}^{U^{k}}, k=1,2,3,4$. The minimax-robust spectral characteristic of the optimal estimate of the functional $A \vec{\xi}$ is determined by the formula (23).

\subsection{Least favourable spectral density in classes of " $\varepsilon$-contaminated" densities}

Consider the prediction problem for the functional $A \vec{\xi}$, which depends on unobserved values of a sequence $\vec{\xi}(m)$, with stationary increments based on observations of the sequence at points $m=-1,-2, \ldots$ under the condition that the sets of admissible spectral densities $\mathcal{D}_{\varepsilon}^{k}$, $k=1,2,3,4$, are defined as follows:

$$
\begin{gathered}
\mathcal{D}_{\varepsilon}^{1}=\left\{f(\lambda): \operatorname{Tr}[f(\lambda)]=\frac{\operatorname{Tr}\left[f_{1}(\lambda)\right]}{(1-\varepsilon)^{-1}}+\varepsilon \operatorname{Tr}[W(\lambda)], \frac{1}{2 \pi} \int_{-\pi}^{\pi} \frac{\left|1-e^{i \lambda \mu}\right|^{2 n}}{|\lambda|^{2 n}} \operatorname{Tr}[f(\lambda)] d \lambda=p\right\}, \\
\mathcal{D}_{\varepsilon}^{2}=\left\{f(\lambda): f_{k k}(\lambda)=\frac{f_{k k}^{1}(\lambda)}{(1-\varepsilon)^{-1}}+\varepsilon w_{k k}(\lambda), \frac{1}{2 \pi} \int_{-\pi}^{\pi} \frac{\left|1-e^{i \lambda \mu}\right|^{2 n}}{|\lambda|^{2 n}} f_{k k}(\lambda) d \lambda=p_{k}, k=\overline{1, T}\right\}, \\
\mathcal{D}_{\varepsilon}^{3}=\left\{f(\lambda):\left\langle B_{1}, f(\lambda)\right\rangle=\frac{\left\langle B_{1}, f_{1}(\lambda)\right\rangle}{(1-\varepsilon)^{-1}}+\varepsilon\left\langle B_{1}, W(\lambda)\right\rangle, \frac{1}{2 \pi} \int_{-\pi}^{\pi} \frac{\left|1-e^{i \lambda \mu}\right|^{2 n}}{|\lambda|^{2 n}}\left\langle B_{1}, f(\lambda)\right\rangle d \lambda=p\right\}, \\
\mathcal{D}_{\varepsilon}^{4}=\left\{f(\lambda): f(\lambda)=(1-\varepsilon) f_{1}(\lambda)+\varepsilon W(\lambda), \frac{1}{2 \pi} \int_{-\pi}^{\pi} \frac{\left|1-e^{i \lambda \mu}\right|^{2 n}}{|\lambda|^{2 n}} f(\lambda) d \lambda=P\right\} .
\end{gathered}
$$

Here $f_{1}(\lambda)$ is a fixed spectral density, $W(\lambda)$ is an unknown spectral density, $p, p_{k}, k=\overline{1, T}$, are given numbers, $P$ is a given positive-definite Hermitian matrices.

From the condition $0 \in \partial \Delta_{\mathcal{D}}\left(f_{0}\right)$ we find the following equations, which determine the least favourable spectral densities for these given sets of admissible spectral densities.

For the first set of admissible spectral densities $\mathcal{D}_{\mathcal{\varepsilon}}^{1}$, we derive equation

$$
\vec{C}_{\mu}^{f 0}\left(e^{i \lambda}\right)\left(\vec{C}_{\mu}^{f 0}\left(e^{i \lambda}\right)\right)^{*}=\left(\alpha^{2}+\gamma_{1}(\lambda)\right)\left(\frac{\left|1-e^{i \lambda \mu}\right|^{2 n}}{|\lambda|^{2 n}} f_{0}(\lambda)\right)^{2},
$$


where $\alpha^{2}$ is Lagrange multiplier, $\gamma_{1}(\lambda) \leq 0$ and $\gamma_{1}(\lambda)=0$ if $\operatorname{Tr}\left[f_{0}(\lambda)\right]>(1-\varepsilon) \operatorname{Tr}\left[f_{1}(\lambda)\right]$.

For the second set of admissible spectral densities $\mathcal{D}_{\varepsilon}^{2}$, we derive equation

$$
\vec{C}_{\mu}^{f 0}\left(e^{i \lambda}\right)\left(\vec{C}_{\mu}^{f 0}\left(e^{i \lambda}\right)\right)^{*}=\frac{\left|1-e^{i \lambda \mu}\right|^{2 n}}{|\lambda|^{2 n}} f_{0}(\lambda)\left\{\left(\alpha_{k}^{2}+\gamma_{k}^{1}(\lambda)\right) \delta_{k l}\right\}_{k, l=1}^{T} \frac{\left|1-e^{i \lambda \mu}\right|^{2 n}}{|\lambda|^{2 n}} f_{0}(\lambda),
$$

where $\alpha_{k}^{2}$ are Lagrange multipliers, $\gamma_{k}^{1}(\lambda) \leq 0$ and $\gamma_{k}^{1}(\lambda)=0$ if $f_{k k}^{0}(\lambda)>(1-\varepsilon) f_{k k}^{1}(\lambda)$.

For the third set of admissible spectral densities $\mathcal{D}_{\varepsilon}^{3}$, we derive equation

$$
\left.\vec{C}_{\mu}^{f 0}\left(e^{i \lambda}\right)\left(\vec{C}_{\mu}^{f 0}\left(e^{i \lambda}\right)\right)^{*}=\left(\alpha^{2}+\gamma_{1}^{\prime}(\lambda)\right) \frac{\left|1-e^{i \lambda \mu}\right|^{2 n}}{|\lambda|^{2 n}} f_{0}(\lambda)\right) B_{1}^{\top} \frac{\left|1-e^{i \lambda \mu}\right|^{2 n}}{|\lambda|^{2 n}} f_{0}(\lambda),
$$

where $\alpha^{2}$ is a Lagrange multiplier, $\gamma_{1}^{\prime}(\lambda) \leq 0$ and $\gamma_{1}^{\prime}(\lambda)=0$ if $\left\langle B_{1}, f_{0}(\lambda)\right\rangle>(1-\varepsilon)\left\langle B_{1}, f_{1}(\lambda)\right\rangle$.

For the fourth set of admissible spectral densities $\mathcal{D}_{\varepsilon}^{4}$, we derive equation

$$
\vec{C}_{\mu}^{f 0}\left(e^{i \lambda}\right)\left(\vec{C}_{\mu}^{f 0}\left(e^{i \lambda}\right)\right)^{*}=\frac{\left|1-e^{i \lambda \mu}\right|^{2 n}}{|\lambda|^{2 n}} f_{0}(\lambda)\left(\vec{\alpha} \cdot \vec{\alpha}^{*}+\Gamma(\lambda)\right) \frac{\left|1-e^{i \lambda \mu}\right|^{2 n}}{|\lambda|^{2 n}} f_{0}(\lambda),
$$

where $\vec{\alpha}$ is a vector of Lagrange multipliers, $\Gamma(\lambda) \leq 0$ and $\Gamma(\lambda)=0$ if $f_{0}(\lambda)>(1-\varepsilon) f_{1}(\lambda)$.

In the case, when the spectral density admits the canonical factorization (22), we have the following equations, correspondingly

$$
\begin{gathered}
\vec{r}_{\mu}^{0}\left(e^{i \lambda}\right)\left(\vec{r}_{\mu}^{0}\left(e^{i \lambda}\right)\right)^{*}=\left(\alpha^{2}+\gamma_{1}(\lambda)\right)\left(\Phi_{\mu}^{0}\left(e^{-i \lambda}\right)\right)^{\top} \overline{\Phi_{\mu}^{0}\left(e^{-i \lambda}\right)}, \\
\vec{r}_{\mu}^{0}\left(e^{i \lambda}\right)\left(\vec{r}_{\mu}^{0}\left(e^{i \lambda}\right)\right)^{*}=\left(\Phi_{\mu}^{0}\left(e^{-i \lambda}\right)\right)^{\top}\left\{\left(\alpha_{k}^{2}+\gamma_{k}^{1}(\lambda)\right) \delta_{k l}\right\}_{k, l=1}^{T} \overline{\Phi_{\mu}^{0}\left(e^{-i \lambda}\right)}, \\
\vec{r}_{\mu}^{0}\left(e^{i \lambda}\right)\left(\vec{r}_{\mu}^{0}\left(e^{i \lambda}\right)\right)^{*}=\left(\alpha^{2}+\gamma_{1}^{\prime}(\lambda)\right)\left(\Phi_{\mu}^{0}\left(e^{-i \lambda}\right)\right)^{\top} B_{1}^{\top} \overline{\Phi_{\mu}^{0}\left(e^{-i \lambda}\right)}, \\
\vec{r}_{\mu}^{0}\left(e^{i \lambda}\right)\left(\vec{r}_{\mu}^{0}\left(e^{i \lambda}\right)\right)^{*}=\left(\Phi_{\mu}^{0}\left(e^{-i \lambda}\right)\right)^{\top}\left(\vec{\alpha} \cdot \vec{\alpha}^{*}+\Gamma(\lambda)\right) \overline{\Phi_{\mu}^{0}\left(e^{-i \lambda}\right)} .
\end{gathered}
$$

The following theorem holds true.

Theorem 11. The least favourable spectral densities $f_{0}(\lambda)$ in the classes $\mathcal{D}_{\mathcal{\varepsilon}}^{k}, k=1,2,3,4$, for the optimal linear extrapolation of the functional $A \vec{\xi}$ from observations of the sequence $\vec{\xi}(m)$ at points $m=-1,-2, \ldots$ are determined by the minimality condition (12), equations (58)(61), respectively, the constrained optimization problem (39) and restrictions on densities from the corresponding classes $\mathcal{D}_{\varepsilon}^{k}, k=1,2,3,4$. The minimax-robust spectral characteristic of the optimal estimate of the functional $A \vec{\xi}$ is determined by the formula (20). In the case, when spectral densities admit the canonical factorization (22), the least favourable spectral densities $f_{0}(\lambda)$ are determined by the minimality condition (12), equations(62)-(65), respectively, the constrained optimization problem (40) and restrictions on densities from the corresponding classes $\mathcal{D}_{\varepsilon}^{k}, k=1,2,3,4$. The minimax-robust spectral characteristic of the optimal estimate of the functional $A \vec{\xi}$ is determined by the formula (23).

\subsection{Least favourable spectral density in classes which describe " $\delta$-neighborhood" models}

Consider the prediction problem for the functional $A \vec{\xi}$, which depends on unobserved values of a sequence $\vec{\xi}(m)$, with stationary increments based on observations of the sequence at points $m=-1,-2, \ldots$ under the condition that the sets of admissible spectral densities $\mathcal{D}_{1 \delta}^{k}$, 
$k=1,2,3,4$, are defined as follows:

$$
\begin{gathered}
\mathcal{D}_{1 \delta}^{1}=\left\{f(\lambda): \frac{1}{2 \pi} \int_{-\pi}^{\pi} \frac{\left|1-e^{i \lambda \mu}\right|^{2 n}}{|\lambda|^{2 n}} \| \operatorname{Tr}\left(f(\lambda)-f_{1}(\lambda)\right) \mid d \lambda \leq \delta\right\}, \\
\mathcal{D}_{1 \delta}^{2}=\left\{f(\lambda): \frac{1}{2 \pi} \int_{-\pi}^{\pi} \frac{\left|1-e^{i \lambda \mu}\right|^{2 n}}{|\lambda|^{2 n}}\left|f_{k k}(\lambda)-f_{k k}^{1}(\lambda)\right| d \lambda \leq \delta_{k}, k=\overline{1, T}\right\}, \\
\mathcal{D}_{1 \delta}^{3}=\left\{f(\lambda): \frac{1}{2 \pi} \int_{-\pi}^{\pi} \frac{\left|1-e^{i \lambda \mu}\right|^{2 n}}{|\lambda|^{2 n}}\left|\left\langle B_{2}, f(\lambda)-f_{1}(\lambda)\right\rangle\right| d \lambda \leq \delta\right\}, \\
\mathcal{D}_{1 \delta}^{4}=\left\{f(\lambda): \frac{1}{2 \pi} \int_{-\pi}^{\pi} \frac{\left|1-e^{i \lambda \mu}\right|^{2 n}}{|\lambda|^{2 n}}\left|f_{i j}(\lambda)-f_{i j}^{1}(\lambda)\right| d \lambda \leq \delta_{i}^{j}, i, j=\overline{1, T}\right\} .
\end{gathered}
$$

Here $f_{1}(\lambda)$ is a fixed spectral density, $\delta, \delta_{k}, k=\overline{1, T}, \delta_{i}^{j}, i, j=\overline{1, T}$, are given numbers.

From the condition $0 \in \partial \Delta_{\mathcal{D}}\left(f_{0}\right)$ we find the following equations, which determine the least favourable spectral densities for these given sets of admissible spectral densities.

For the first set of admissible spectral densities $\mathcal{D}_{1 \delta}^{1}$, we derive equations

$$
\begin{gathered}
\vec{C}_{\mu}^{f 0}\left(e^{i \lambda}\right)\left(\vec{C}_{\mu}^{f 0}\left(e^{i \lambda}\right)\right)^{*}=\beta^{2} \gamma_{2}(\lambda)\left(\frac{\left|1-e^{i \lambda \mu}\right|^{2 n}}{|\lambda|^{2 n}} f_{0}(\lambda)\right)^{2}, \\
\frac{1}{2 \pi} \int_{-\pi}^{\pi} \frac{\left|1-e^{i \lambda \mu}\right|^{2 n}}{|\lambda|^{2 n}}\left|\operatorname{Tr}\left(f_{0}(\lambda)-f_{1}(\lambda)\right)\right| d \lambda=\delta,
\end{gathered}
$$

where $\beta^{2}$ is Lagrange multiplier, $\left|\gamma_{2}(\lambda)\right| \leq 1, \gamma_{2}(\lambda)=\operatorname{sign}\left(\operatorname{Tr}\left(f_{0}(\lambda)-f_{1}(\lambda)\right)\right): \operatorname{Tr}\left(f_{0}(\lambda)-\right.$ $\left.f_{1}(\lambda)\right) \neq 0$.

For the second set of admissible spectral densities $\mathcal{D}_{1 \delta}^{2}$, we derive equations

$$
\begin{gathered}
\vec{C}_{\mu}^{f 0}\left(e^{i \lambda}\right)\left(\vec{C}_{\mu}^{f 0}\left(e^{i \lambda}\right)\right)^{*}=\frac{\left|1-e^{i \lambda \mu}\right|^{2 n}}{|\lambda|^{2 n}} f_{0}(\lambda)\left\{\beta_{k}^{2} \gamma_{k}^{2}(\lambda) \delta_{k l}\right\}_{k, l=1}^{T} \frac{\left|1-e^{i \lambda \mu}\right|^{2 n}}{|\lambda|^{2 n}} f_{0}(\lambda), \\
\frac{1}{2 \pi} \int_{-\pi}^{\pi} \frac{\left|1-e^{i \lambda \mu}\right|^{2 n}}{|\lambda|^{2 n}}\left|f_{k k}^{0}(\lambda)-f_{k k}^{1}(\lambda)\right| d \lambda=\delta_{k}
\end{gathered}
$$

where $\beta_{k}^{2}$ are Lagrange multipliers, $\left|\gamma_{k}^{2}(\lambda)\right| \leq 1$ and $\gamma_{k}^{2}(\lambda)=\operatorname{sign}\left(f_{k k}^{0}(\lambda)-f_{k k}^{1}(\lambda)\right): f_{k k}^{0}(\lambda)-$ $f_{k k}^{1}(\lambda) \neq 0, k=\overline{1, T}$.

For the third set of admissible spectral densities $\mathcal{D}_{1 \delta}^{3}$, we derive equations

$$
\begin{gathered}
\vec{C}_{\mu}^{f 0}\left(e^{i \lambda}\right)\left(\vec{C}_{\mu}^{f 0}\left(e^{i \lambda}\right)\right)^{*}=\beta^{2} \gamma_{2}^{\prime}(\lambda) \frac{\left|1-e^{i \lambda \mu}\right|^{2 n}}{|\lambda|^{2 n}} f_{0}(\lambda) B_{2}^{\top} \frac{\left|1-e^{i \lambda \mu}\right|^{2 n}}{|\lambda|^{2 n}} f_{0}(\lambda), \\
\frac{1}{2 \pi} \int_{-\pi}^{\pi} \frac{\left|1-e^{i \lambda \mu}\right|^{2 n}}{|\lambda|^{2 n}}\left|\left\langle B_{2}, f_{0}(\lambda)-f_{1}(\lambda)\right\rangle\right| d \lambda=\delta,
\end{gathered}
$$

where $\beta^{2}$ is a Lagrange multiplier, $\left|\gamma_{2}^{\prime}(\lambda)\right| \leq 1, \gamma_{2}^{\prime}(\lambda)=\operatorname{sign}\left\langle B_{2}, f_{0}(\lambda)-f_{1}(\lambda)\right\rangle:\left\langle B_{2}, f_{0}(\lambda)-\right.$ $\left.f_{1}(\lambda)\right\rangle \neq 0$.

For the fourth set of admissible spectral densities $\mathcal{D}_{1 \delta}^{4}$, we derive equations

$$
\begin{gathered}
\vec{C}_{\mu}^{f 0}\left(e^{i \lambda}\right)\left(\vec{C}_{\mu}^{f 0}\left(e^{i \lambda}\right)\right)^{*}=\frac{\left|1-e^{i \lambda \mu}\right|^{2 n}}{|\lambda|^{2 n}} f_{0}(\lambda)\left\{\beta_{i j}(\lambda) \gamma_{i j}(\lambda)\right\}_{i, j=1}^{T} \frac{\left|1-e^{i \lambda \mu}\right|^{2 n}}{|\lambda|^{2 n}} f_{0}(\lambda), \\
\frac{1}{2 \pi} \int_{-\pi}^{\pi} \frac{\left|1-e^{i \lambda \mu}\right|^{2 n}}{|\lambda|^{2 n}}\left|f_{i j}^{0}(\lambda)-f_{i j}^{1}(\lambda)\right| d \lambda=\delta_{i}^{j},
\end{gathered}
$$


where $\beta_{i j}$ are Lagrange multipliers, $\left|\gamma_{i j}(\lambda)\right| \leq 1, \gamma_{i j}(\lambda)=\left(f_{i j}^{0}(\lambda)-f_{i j}^{1}(\lambda)\right) /\left|f_{i j}^{0}(\lambda)-f_{i j}^{1}(\lambda)\right|$ : $f_{i j}^{0}(\lambda)-f_{i j}^{1}(\lambda) \neq 0, i, j=\overline{1, T}$.

In the case, when the spectral density admits the canonical factorization (22), we have the following equations, correspondingly

$$
\begin{gathered}
\vec{r}_{\mu}^{0}\left(e^{i \lambda}\right)\left(\vec{r}_{\mu}^{0}\left(e^{i \lambda}\right)\right)^{*}=\beta^{2} \gamma_{2}(\lambda)\left(\Phi_{\mu}^{0}\left(e^{-i \lambda}\right)\right)^{\top} \overline{\Phi_{\mu}^{0}\left(e^{-i \lambda}\right)}, \\
\vec{r}_{\mu}^{0}\left(e^{i \lambda}\right)\left(\vec{r}_{\mu}^{0}\left(e^{i \lambda}\right)\right)^{*}=\left(\Phi_{\mu}^{0}\left(e^{-i \lambda}\right)\right)^{\top}\left\{\beta_{k}^{2} \gamma_{k}^{2}(\lambda) \delta_{k l}\right\}_{k, l=1}^{T} \overline{\Phi_{\mu}^{0}\left(e^{-i \lambda}\right)} \\
\vec{r}_{\mu}^{0}\left(e^{i \lambda}\right)\left(\vec{r}_{\mu}^{0}\left(e^{i \lambda}\right)\right)^{*}=\beta^{2} \gamma_{2}^{\prime}(\lambda)\left(\Phi_{\mu}^{0}\left(e^{-i \lambda}\right)\right)^{\top} B_{2}^{\top} \overline{\Phi_{\mu}^{0}\left(e^{-i \lambda}\right)}, \\
\vec{r}_{\mu}^{0}\left(e^{i \lambda}\right)\left(\vec{r}_{\mu}^{0}\left(e^{i \lambda}\right)\right)^{*}=\left(\Phi_{\mu}^{0}\left(e^{-i \lambda}\right)\right)^{\top}\left\{\beta_{i j}(\lambda) \gamma_{i j}(\lambda)\right\}_{i, j=1}^{T} \overline{\Phi_{\mu}^{0}\left(e^{-i \lambda}\right)} .
\end{gathered}
$$

The following theorem holds true.

Theorem 12. The least favourable spectral densities $f_{0}(\lambda)$ in the classes $\mathcal{D}_{1 \delta}^{k}, k=1,2,3,4$, for the optimal linear extrapolation of the functional $A \vec{\xi}$ from observations of the sequence $\vec{\xi}(m)$ at points $m=-1,-2, \ldots$ are determined by the minimality condition (12), equations (66)(73), respectively, the constrained optimization problem (39) and restrictions on densities from the corresponding classes $\mathcal{D}_{1 \delta}^{k}, k=1,2,3,4$. The minimax-robust spectral characteristic of the optimal estimate of the functional $A \vec{\xi}$ is determined by the formula 20. In the case, when spectral densities admit the canonical factorization (22), the least favourable spectral densities $f_{0}(\lambda)$ are determined by the minimality condition (12), equations (74)-(77), respectively, the constrained optimization problem (40), and restrictions on densities from the corresponding classes $\mathcal{D}_{1 \delta}^{k}, k=1,2,3,4$. The minimax-robust spectral characteristic of the optimal estimate of the functional $A \vec{\xi}$ is determined by the formula (23).

\section{Conclusions}

In this article, we present results of investigation of stochastic sequences with periodically stationary increments. We give definition of the increment sequence and introduce stochastic sequences with periodically stationary (periodically correlated, cyclostationary) increments. These non-stationary stochastic sequences combine periodic structure of covariation functions of sequences as well as integrating one. A short review of the spectral theory of vector-valued increment sequences is presented.

We describe methods of solution of the forecasting problem for linear functionals, which are constructed from unobserved values of a sequence with periodically stationary increments. Estimates are obtained by representing the sequence under investigation as a vector-valued sequence with stationary increments. The problem is investigated in the case of spectral certainty, where the spectral density of the sequence is exactly known. In this case, we propose an approach based on the Hilbert space projection method. We derive formulas for calculating the spectral characteristics and the mean-square errors of the optimal estimates of the functionals. In the case of spectral uncertainty, where the spectral density is not exactly known while, instead, a set of admissible spectral densities is specified, the minimax-robust method is applied. We propose a representation of the mean square error in the form of a linear functional in $L_{1}$ with respect to spectral densities, which allows us to solve the corresponding constrained optimization problem and describe the minimax (robust) estimates of the functionals. Formulas 
that determine the least favourable spectral densities and minimax (robust) spectral characteristic of the optimal linear estimates of the functionals are derived for a collection of specific classes of admissible spectral densities.

These least favourable spectral density matrices are solutions of the optimization problem $\Delta_{D}(f)=-\Delta\left(h\left(f_{0}\right) ; f\right)+\delta(f \mid D) \rightarrow$ inf, which is characterized by the condition $0 \in \partial \Delta_{D}\left(f_{0}\right)$, where $\partial \Delta_{D}\left(f_{0}\right)$ is the subdifferential of the convex functional $\Delta_{D}(f)$ at point $f_{0}$. The form of the functional $\Delta\left(h\left(f_{0}\right) ; f\right)$ is convenient for application of the Lagrange method of indefinite multipliers for finding solution to the optimization problem. The complexity of solution of the problem is determined by the complexity of calculating of subdifferentials of the indicator functions $\delta(f \mid D)$ of sets $D$. Making use of the method of Lagrange multipliers and the form of subdifferentials of the indicator functions, we describe relations that determine the least favourable spectral densities in some special classes of spectral densities. These are: classes $D_{0}$ of densities with the integral restrictions, classes $D_{1 \delta}$, which describe the " $\delta$-neighborhood" models in the space $L_{1}$ of a fixed bounded spectral density, classes $D_{\varepsilon}$, which describe the " $\varepsilon$-contaminated" models of a fixed bounded spectral density, classes $D_{V}^{U}$, which describe the "strip" models of spectral densities.

\section{References}

[1] Baek C., Davis R.A., Pipiras V. Periodic dynamic factor models: estimation approaches and applications. Electron. J. Stat. 2018, 12 (2), 4377-4411. doi:10.1214/18-EJS1518

[2] Basawa I.V., Lund R., Shao Q. First-order seasonal autoregressive processes with periodically varying parameters. Statist. Probab. Lett. 2004, 67 (4), 299-306. doi:10.1016/j.spl.2004.02.001

[3] Box G.E.P., Jenkins G.M., Reinsel G.C., Ljung G.M. Time series analysis: forecasting and control. 5rd ed., John Wiley \& Sons, Hoboken, 2016.

[4] Box G.E.P., Jenkins G.M. Time series analysis: forecasting and control. Holden-Day, San Francisco, 1970.

[5] Dubovets'ka I.I., Moklyachuk M.P. Extrapolation of periodically correlated processes from observations with noise. Theory Probab. Math. Statist. 2014, 88, 67-83. doi:10.1090/S0094-9000-2014-00919-9

[6] Dudek A., Hurd H., Wojtowicz W. Periodic autoregressive moving average methods based on Fourier representation of periodic coefficients. Wiley Interdiscip. Rev. Comput. Stat. 2016, 8 (3), 130-149. doi:10.1002/wics.1380

[7] Franke J. Minimax-robust prediction of discrete time series. Z. Wahrscheinlichkeitstheor. Verw. Geb. 1985, 68 (3), 337-364. doi:10.1007/BF00532645

[8] Gikhman I.I., Skorokhod A.V. Introduction to the theory of random processes. Fizmatlit, Moscow, 1965. (in Russian)

[9] Gladyshev E.G. Periodically correlated random sequences. Dokl. Akad. Nauk SSSR 1961, 137 (5), 1026-1029. (in Russian)

[10] Grenander U. A prediction problem in game theory. Ark. Mat. 1957, 3 (4), 371-379. doi:10.1007/BF02589429

[11] Hannan E.J. Multiple time series. John Wiley \& Sons, New York, 1970.

[12] Hosoya Y. Robust linear extrapolations of second-order stationary processes. Ann. Probab. 1978, 6 (4), 574-584. doi:10.1214/aop/1176995479

[13] Johansen S., Nielsen M.O. The role of initial values in conditional sum-of-squares estimation of nonstationary fractional time series models. Econometric Theory 2016, 32 (5), 1095-1139. doi:10.1017/S0266466615000110

[14] Karhunen K. Über lineare methoden in der wahrscheinlichkeitsrechnung. Suomalainen Tiedeakatemia, Helsinki, 1947. 
[15] Kassam S.A. Robust hypothesis testing and robust time series interpolation and regression. J. Time Series Anal. 1982, 3 (3), 185-194. doi:10.1111/j.1467-9892.1982.tb00341.x

[16] Kassam S.A., Poor H.V. Robust techniques for signal processing: A survey. Proc. IEEE 1985, 73 (3), $433-481$. doi:10.1109/PROC.1985.13167

[17] Kolmogorov A.N. Selected works of A.N. Kolmogorov. Volume II Probability theory and mathematical statistics. In: Shiryayev A.N. (Ed.) Mathematics and its Applications, 26. Kluwer, Dordrecht, 1992.

[18] Kozak P.S., Moklyachuk M.P. Estimates of functionals constructed from random sequences with periodically stationary increments. Theory Probab. Math. Statist. 2018, 97, 85-98. doi:10.1090/tpms/1050

[19] Lund R. Choosing seasonal autocovariance structures: PARMA or SARMA? In: Bell W.R., Holan S.H., McElroy T.S. (Eds.) Economic time series: modelling and seasonality. 2011, Chapman and Hall, London.

[20] Luz M., Moklyachuk M. Minimax-robust prediction problem for stochastic sequences with stationary increments and cointegrated sequences. Stat. Optim. Inf. Comput. 2015, 3 (2), 160-188. doi:10.19139/soic.v3i2.132

[21] Luz M., Moklyachuk M. Estimation of stochastic processes with stationary increments and cointegrated sequences. John Wiley \& Sons, New York, 2019.

[22] Masyutka O.Yu., Moklyachuk M.P., Sidei M.I. Filtering of multidimensional stationary sequences with missing observations. Carpathian Math. Publ. 2019, 11 (2), 361-378. doi:10.15330/cmp.11.2.361-378

[23] Moklyachuk M.P. Minimax extrapolation and autoregressive-moving average processes. Theory Probab. Math. Statist. 1990, 41, 77-84.

[24] Moklyachuk M.P. Minimax-robust estimation problems for stationary stochastic sequences. Stat. Optim. Inf. Comput. 2015, 3 (4), 348-419. doi:10.19139/soic.v3i4.173

[25] Moklyachuk M.P., Masyutka A.Yu. Extrapolation of multidimensional stationary processes. Random Oper. Stoch. Equ. 2006, 14 (3), 233-244. doi:10.1515/156939706778239819

[26] Moklyachuk M.P., Masyutka A.Yu. Minimax prediction problem for multidimensional stationary stochastic processes. Comm. Statist. Theory Methods 2011, 40 (19-20), 3700-3710. doi:10.1080/03610926.2011.581190

[27] Moklyachuk M.P., Sidei M.I. Extrapolation problem for stationary sequences with missing observations. Stat., Optim. Inf. Comput. 2017, 5 (3), 212-233. doi:10.19139/soic.v5i3.284

[28] Moklyachuk M.P., Sidei M.I., Masyutka O.Yu. Estimation of stochastic processes with missing observations. Nova Science Publishers, New York, 2019.

[29] Napolitano A. Cyclostationarity: new trends and applications. Signal Process. 2016, 120, 385-408. doi: 10.1016/j.sigpro.2015.09.011

[30] Osborn D. The implications of periodically varying coefficients for seasonal time-series processes. J. Econometrics 1991, 48 (3), 373-384. doi:10.1016/0304-4076(91)90069-P

[31] Pinsker M.S., Yaglom A.M. On linear extrapolaion of random processes with nth stationary incremens. Dokl. Akad. Nauk SSSR. 1954, 94, 385-388. (in Russian)

[32] Porter-Hudak S. An application of the seasonal fractionally differenced model to the monetary aggegrates. J. Amer. Statist. Assoc. 1990, 85 (410), 338-344. doi:10.1080/01621459.1990.10476206

[33] Reisen V.A., Zamprogno B., Palma W., Arteche J. A semiparametric approach to estimate two seasonal fractional parameters in the SARFIMA model. Math. Comput. Simulation 2014, 98, 1-17. doi:10.1016/j.matcom.2013.11.001

[34] Reisen V.A., Monte E.Z., Franco G.C., Sgrancio A.M., Molinares F.A.F., Bondond P., Ziegelmann F.A., Abraham B. Robust estimation of fractional seasonal processes: modeling and forecasting daily average $\mathrm{SO}_{2}$ concentrations. Math. Comput. Simulation 2018, 146, 27-43. doi:10.1016/j.matcom.2017.10.004

[35] Rockafellar R.T. Convex Analysis. Princeton University Press, Princeton, 1997.

[36] Solci C.C., Reisen V.A., Sarnaglia A.J.Q., Bondon P. Empirical study of robust estimation methods for PAR models with application to the air quality area. Comm. Statist. Theory Methods 2020, 49 (1), 152-168. doi:10.1080/03610926.2018.1533970 
[37] Vastola S.K., Poor H.V. Robust Wiener-Kolmogorov theory. IEEE Trans. Inform. Theory 1984, 30 (2), $316-327$. doi:10.1109/TIT.1984.1056875

[38] Yaglom A.M. Correlation theory of stationary and related random processes with stationary $n$th increments. In: American Mathematical Society Translations: Series 2, 8. American Mathematical Society, Providence, 1958, 87-141. (translation of Mat. Sb. 1955, 37 (79), 141-196. (in Russian))

[39] Yaglom A.M. Correlation theory of stationary and related random functions. Volume I: Basic results, Volume II: Supplementary notes and references. Springer-Verlag, New York, 1987.

Received 06.06.2020

Revised 29.03.2021

Козак П.С., Ауз М.М., Моклячук М.П. Мінімаксний прогноз послідовностей із періодично стаціонарними приростами // Карпатські матем. публ. - 2021. - Т.13, №2. - C. 352-376.

Аосліджується задача оптимального в середньоквадратичному сенсі оцінювання лінійних функціоналів, що залежать від невідомих значень стохастичної послідовності, із періодично стаціонарними приростами за спостереженнями послідовності в точках $k<0$. Знайдено формули для обчислення середньоквадратичних похибок та спектральних характеристик оптимальних оцінок функціоналів у тому випадку, коли спектральна щільність послідовності точно відома. Мінімаксний (робастний) метод оцінювання застосовано у тому випадку, коли спектральна шільність послідовностей точно невідомі, а задані множини допустимих спектральних щільностей. Формули, шо визначають найменш сприятливі спектральні шільністі та мі-

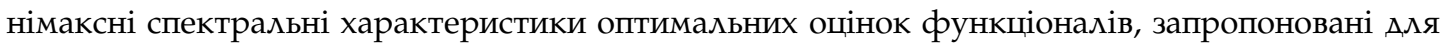
заданих множин допустимих спектральних шільностей.

Ключові слова і фрази: послідовність із періодично стаціонарними приростами, мінімаксна оцінка, робастна оцінка, середньоквадратична похибка, найменш сприятлива спектральна щільність, мінімаксна спектральна характеристика. 